\title{
Convergence of iterated Aluthge transform sequence for diagonalizable matrices II: $\lambda$-Aluthge transform
}

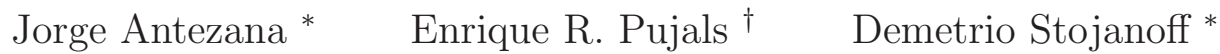

\author{
Jorge Antezana and Demetrio Stojanoff \\ Depto. de Matemática, FCE-UNLP, La Plata, Argentina and IAM-CONICET \\ e-mail: antezana@mate.unlp.edu.ar and demetrio@mate.unlp.edu.ar
}

\section{Enrique R. Pujals}

Instituto Nacional de Matemática Pura y Aplicada (IMPA), Rio de Janeiro, Brasil. e-mail: enrique@impa.br

AMS Subject Classifications: Primary 37D10; Secondary 15A60.

Keywords: Aluthge transform, stable manifold theorem, similarity orbit, polar decomposition.

\begin{abstract}
Let $\lambda \in(0,1)$ and let $T$ be a $r \times r$ complex matrix with polar decomposition $T=U|T|$. Then, the $\lambda$ - Aluthge transform is defined by

$$
\Delta_{\lambda}(T)=|T|^{\lambda} U|T|^{1-\lambda} .
$$

Let $\Delta_{\lambda}^{n}(T)$ denote the n-times iterated Aluthge transform of $T, n \in \mathbb{N}$. We prove that the sequence $\left\{\Delta_{\lambda}^{n}(T)\right\}_{n \in \mathbb{N}}$ converges for every $r \times r$ diagonalizable matrix $T$. We show regularity results for the two parameter map $(\lambda, T) \mapsto \Delta_{\lambda}^{\infty}(T)$, and we study for which matrices the map $(0,1) \ni \lambda \mapsto \Delta_{\lambda}^{\infty}(T)$ is constant.
\end{abstract}

\footnotetext{
** Partially supported by CONICET (PIP 4463/96), Universidad de La Plata (UNLP 11 X472) and ANPCYT (PICT03-09521).

${ }^{\dagger}$ Partially supported by $\mathrm{CNPq}$
} 


\section{Introduction.}

Let $\mathcal{H}$ be a complex Hilbert space, and let $L(\mathcal{H})$ be the algebra of bounded linear operators on $\mathcal{H}$. Given $T \in L(\mathcal{H})$, consider its (left) polar decomposition $T=U|T|$. In order to study the relationship among p-hyponormal operators, Aluthge introduced in [1] the transformation $\Delta_{1 / 2}(\cdot): L(\mathcal{H}) \rightarrow L(\mathcal{H})$ defined by

$$
\Delta_{1 / 2}(T)=|T|^{1 / 2} U|T|^{1 / 2} .
$$

Later on, this transformation, now called Aluthge transform, was also studied in other contexts by several authors, such as Jung, Ko and Pearcy [14] and [15], Ando [2], Ando and Yamazaki [3], Yamazaki [20], Okubo [16] and Wu [19] among others.

In this paper, given $\lambda \in(0,1)$ and $T \in L(\mathcal{H})$, we study the so-called $\lambda$-Aluthge transform of $T$ defined by

$$
\Delta_{\lambda}(T)=|T|^{\lambda} U|T|^{1-\lambda} .
$$

This notion has already been considered by Okubo in [16] (see also [4] and [13]). We denote by $\Delta_{\lambda}^{n}(T)$ the n-times iterated $\lambda$-Aluthge transform of $T$, i.e.

$$
\Delta_{\lambda}^{0}(T)=T ; \quad \text { and } \quad \Delta_{\lambda}^{n}(T)=\Delta_{\lambda}\left(\Delta_{\lambda}^{n-1}(T)\right) \quad n \in \mathbb{N} .
$$

In a previous paper [ [5], we show that the iterates of usual Aluthge transform $\Delta_{1 / 2}^{n}(T)$ converge to a normal matrix $\Delta_{1 / 2}^{\infty}(T)$ for every diagonalizable matrix $T \in \mathcal{M}_{r}(\mathbb{C})$ (of any size). We also proved in [5] the smoothness of the map $T \mapsto \Delta_{1 / 2}^{\infty}(T)$ when it is restricted to a similarity orbit, or to the (open and dense) set $\mathcal{D}_{r}^{*}(\mathbb{C})$ of invertible $r \times r$ matrices with $r$ different eigenvalues. The key idea was to use a dynamical systems approach to the Aluthge transform, thought as acting on the similarity orbit of a diagonal invertible matrix. Recently, Huajun Huang and Tin-Yau Tam [13] showed, with other approach, that the iterates of every $\lambda$-Aluthge transform $\Delta_{\lambda}^{n}(T)$ converge, for every matrix $T \in \mathcal{M}_{r}(\mathbb{C})$ with all its eigenvalues of different moduli.

In this paper, we study the general case of $\lambda$-Aluthge transforms by means of a dynamical systems approach. This allows us to generalize Huajun Huang and Tin-Yau Tam result for every diagonalizable matrix $T \in \mathcal{M}_{r}(\mathbb{C})$, as well as to show regularity results for the two parameter $\operatorname{map}(\lambda, T) \mapsto \Delta_{\lambda}^{\infty}(T)=\lim _{n \in \mathbb{N}} \Delta_{\lambda}^{n}(T)$.

Now we briefly describe the dynamical point of view of the problem: For every $\lambda \in(0,1)$ and any invertible matrix $T$, it holds that $\Delta_{\lambda}(T)=|T|^{\lambda} T|T|^{-\lambda}$. So the $\lambda$-Aluthge transform of $T$ belongs to the similarity orbit of $T$. This suggests that we can study the Aluthge transform restricted to the similarity orbit of some invertible operator. From that point of view, the diagonalizable case has a better dynamical behavior, as detailed in [5]. If $T$ is diagonalizable, the similarity orbit of $T$ coincides with the similarity orbit of some diagonal operator $D$, which we denote $\mathcal{S}(D)$. The unitary orbit of $D$, denoted by $\mathcal{U}(D)$, is a compact submanifold of $\mathcal{S}(D)$ that consists of all normal matrices in $\mathcal{S}(D)$. Hence $\mathcal{U}(D)$ is fixed by the Aluthge transform and, as it was shown in [4, all the limit points of the sequence $\left\{\Delta_{\lambda}^{n}(T)\right\}_{n \in \mathbb{N}}$ belong to $\mathcal{U}(D)$.

As it was shown in [5] for $\lambda=1 / 2$, we show that for any $N \in \mathcal{U}(D)$ there is a local submanifold $\mathcal{W}_{\lambda, N}^{s}$ transversal to $\mathcal{U}(D)$ characterized as the set the matrices (near $N$ ) that converges with a exponential rate to $N$ by the iteration of the $\lambda$-Aluthge transform. Moreover, the union of these submanifolds form an open neighborhood of $\mathcal{U}(D)$ (see Corollary 3.2.2). Thus, 
since the sequence $\left\{\Delta_{\lambda}^{n}(T)\right\}_{n \in \mathbb{N}}$ goes toward $\mathcal{U}(D)$, for some $n_{0}$ large enough the sequence of iterated Aluthge transforms gets into this open neighborhood and converge exponentially.

These results follow from the classical arguments of stable manifolds (first introduced independently by Hadamard and Perron, see Theorem 2.1.3. for details and general results about the stable manifold theorem, see [1] or the Appendix of [5]). In order to use the stable manifold theorem, we show previously that the derivative of the $\lambda$-Aluthge transform in any $N \in \mathcal{U}(D)$ has two invariant complementary directions, one tangent to $\mathcal{U}(D)$, and other one transversal to it where the derivative is a contraction (see Theorem 3.2.1). Using these techniques we prove that $\Delta_{\lambda}^{n}(T) \underset{n \rightarrow \infty}{\longrightarrow} \Delta_{\lambda}^{\infty}(T) \in \mathcal{U}(r)$, for every $r \times r$ diagonalizable matrix $T$. We also prove that the two parameter map $\Delta_{\infty}(\lambda, T)=\Delta_{\lambda}^{\infty}(T)$ is of class $C^{\infty}$, when restricted to $(0,1) \times \mathcal{D}_{r}^{*}(\mathbb{C})$ and to $(0,1) \times \mathcal{S}(D)$ for a diagonal matrix $D \in \mathcal{M}_{r}(\mathbb{C})$.

We also study for which matrices $T$ the map $(0,1) \ni \lambda \mapsto R_{T}(\lambda)=\Delta_{\lambda}^{\infty}(T)$ is constant. Some partial results are obtained, in particular that $R_{T}$ is not constant for most diagonalizable matrices $T$. We also show that $R_{T}$ is constant for every $T$ in the similarity orbit of a diagonal matrix $D$ if $\sigma(D)=\left\{d_{1}, d_{2}\right\}$ with $\left|d_{1}\right|=\left|d_{2}\right|$. We state and discuss the following conjecture: this is the unique case where $R_{T}$ is constant for every $T$ in the similarity orbit of $D$.

The paper has a structure very similar to [5] because, at any step of the dynamical systems argument, we need to state results which differ slightly of those results of [5]. The proofs of these results are omitted or just sketched. The paper is organized as follows: in section 2, we collect several preliminary definitions and results about the the stable manifold theorem, about the geometry of similarity and unitary orbits, and about known results on $\lambda$-Aluthge transforms. In section 3 , we prove the convergence results. In section 4 we study the smoothness of the two parameter map $(\lambda, T) \mapsto \Delta_{\lambda}^{\infty}(T)$ and we study the behavior of the limit function $\Delta_{\lambda}^{\infty}(T)$ with respect to the parameter $\lambda$. The basic tool for these results, in order to apply the stable manifold theorem to the similarity orbit of a diagonal matrix, is the mentioned Theorem 3.2.1. whose proof, somewhat technical, is done in section 5 .

\section{Preliminaries.}

In this paper $\mathcal{M}_{r}(\mathbb{C})$ denotes the algebra of complex $r \times r$ matrices, $\mathcal{G} l_{r}(\mathbb{C})$ the group of all invertible elements of $\mathcal{M}_{r}(\mathbb{C}), \mathcal{U}(r)$ the group of unitary operators, and $\mathcal{M}_{r}^{h}(\mathbb{C})\left(\right.$ resp. $\left.\mathcal{M}_{r}^{a h}(\mathbb{C})\right)$ denotes the real algebra of Hermitian (resp. anti Hermitian) matrices. Given $T \in \mathcal{M}_{r}(\mathbb{C})$, $R(T)$ denotes the range or image of $T$, $\operatorname{ker} T$ the null space of $T, \sigma(T)$ the spectrum of $T$, rk $\mathrm{T}=\operatorname{dim} R(T)$ the rank of $T, \operatorname{tr}(T)$ the trace of $T$, and $T^{*}$ the adjoint of $T$. If $v \in \mathbb{C}^{r}$, we denote by $\operatorname{diag}(v) \in \mathcal{M}_{r}(\mathbb{C})$ the diagonal matrix with $v$ in its diagonal. We shall consider the space of matrices $\mathcal{M}_{r}(\mathbb{C})$ as a real Hilbert space with the inner product defined by

$$
\langle A, B\rangle=\mathbb{R e}\left(\operatorname{tr}\left(B^{*} A\right)\right) .
$$

The norm induced by this inner product is the so-called Frobenius norm, denoted by $\|\cdot\|_{2}$.

On the other hand, let $M$ be a manifold. By means of $T M$ we denote the tangent bundle of $M$ and by means of $T_{x} M$ we denote the tangent space at the point $x \in M$. Given a function $f \in C^{r}(M)$, where $r=1, \ldots, \infty, T_{x} f(v)$ denotes the derivative of $f$ at the point $x$ applied to the vector $v$. 


\subsection{Stable manifold theorem}

In this section we state the stable manifold theorem for an invariant set of a smooth endomorphism (see 2.1.3 below). We refer to [5] for a detailed description of these type of results. Let $M$ be a smooth Riemann manifold and $N \subseteq M$ a submanifold (not necessarily compact). Throughout this subsection $T_{N} M$ denotes the tangent bundle of $M$ restricted to $N$.

Definition 2.1.1. A $C^{r}$ pre-lamination indexed by $N$ is a continuous choice of a $C^{r}$ embedded disc $\mathcal{B}_{x}$ through each $x \in N$. Continuity means that $N$ is covered by open sets $\mathcal{U}$ in which $x \rightarrow B_{x}$ is given by

$$
\mathcal{B}_{x}=\sigma(x)\left((-\varepsilon, \varepsilon)^{k}\right)
$$

where $\sigma: \mathcal{U} \cap N \rightarrow \operatorname{Emb}^{r}\left((-\varepsilon, \varepsilon)^{k}, M\right)$ is a continuous section. Note that $\operatorname{Emb}^{r}\left((-\varepsilon, \varepsilon)^{k}, M\right)$ is a $C^{r}$ fiber bundle over $M$ whose projection is $\beta \rightarrow \beta(0)$. Thus $\sigma(x)(0)=x$. If the sections mentioned above are $C^{s}, 1 \leq s \leq r$, we say that the $C^{r}$ pre-lamination is of class $C^{s}$.

Definition 2.1.2. Let $f$ be a smooth endomorphism of $M, \rho>0$, and suppose that $\left.f\right|_{N}$ is a homeomorphism. Then, $N$ is $\rho$-pseudo hyperbolic for $f$ if there exist two smooth subbundles of $T_{N} M$, denoted by $\mathcal{E}^{s}$ and $\mathcal{F}$, such that

1. $T_{N} M=\mathcal{E}^{s} \oplus \mathcal{F}$

2. $T N=\mathcal{F}$

3. Both, $\mathcal{E}^{s}$ and $\mathcal{F}$, are $T f$-invariant;

4. $T f$ restricted to $\mathcal{F}$ is an automorphism, which expand it by a factor greater than $\rho$.

5. $T_{x} f: \mathcal{E}_{x}^{s} \rightarrow \mathcal{E}_{f(x)}^{s}$ has norm lower than $\rho$.

In this case, the stable manifold theorem assures that for any point $x \in N$ it is possible to find an $f$-invariant submanifold transversal to $N$ tangent to $\mathcal{E}^{s}$ and characterized as the set of points with trajectories asymptotic to the trajectory of $x$. We shall state the following particular version of this theorem. For a proof, see Theorem 2.1.4, Corollary 2.1.5 and Remark 2.1.6 of [5].

Theorem 2.1.3 (Stable manifold theorem for a submanifold of fixed points). Let $f$ be a $C^{r}$ endomorphism of $M$ with a $\rho$-pseudo hyperbolic submanifold $N$ with $\rho<1$. Assume that any point $p$ in $N$ is a fixed point. Then, there is a $f$-invariant $C^{r}$-pre-lamination $\mathcal{W}^{s}: N \rightarrow$ $\operatorname{Emb}^{r}\left((-1,1)^{k}, M\right)$ of class $\mathbf{C}^{\mathbf{r}}$ such that, for every $x \in N$,

1. $\mathcal{W}^{s}(x)(0)=x$

2. $\mathcal{W}_{x}^{s}=\mathcal{W}^{s}(x)\left((-1,1)^{k}\right)$ is tangent to $\mathcal{E}_{x}^{s}$ at every $x \in N$,

3. There exists an open neighborhood $\mathcal{U}$ of $x$ (open relative to $M$ ) such that

$$
\mathcal{W}_{x}^{s} \cap \mathcal{U}=\left\{y \in \mathcal{U}: \operatorname{dist}\left(x, f^{n}(y)\right)<\operatorname{dist}(x, y) \rho^{n}\right\} .
$$

4. If $y \in N, x \neq y$, then $\mathcal{W}_{x}^{s} \cap \mathcal{W}_{y}^{s}=\varnothing$. 
5. There exists $\gamma>0$ such that

$$
B(x, \gamma) \subset \bigcup_{x \in N} \mathcal{W}_{x}^{s}
$$

This implies that $\bigcup_{x \in N} \mathcal{W}_{x}^{s}$ contains an open neighborhood $\mathcal{W}(N)$ of $N$ in $M$.

6. The map

$$
p: \mathcal{W}(N) \rightarrow N \quad \text { given by } \quad p(a)=x \quad \text { if } \quad a \in \mathcal{W}_{x}^{s}(x),
$$

is well defined and it is of class $C^{r}$.

\subsection{Similarity orbit of a diagonal matrix}

In this subsection we recall some facts about the similarity orbit of a diagonal matrix.

Definition 2.2.1. Let $D \in \mathcal{M}_{r}(\mathbb{C})$ be diagonal. The similarity orbit of $D$ is the set

$$
\mathcal{S}(D)=\left\{S D S^{-1}: S \in \mathcal{G} l_{r}(\mathbb{C})\right\} .
$$

On the other hand, $\mathcal{U}(D)=\left\{U D U^{*}: U \in \mathcal{U}(r)\right\}$ denotes the unitary orbit of $D$. We donote by $\pi_{D}: \mathcal{G} l_{r}(\mathbb{C}) \rightarrow \mathcal{S}(D) \subseteq \mathcal{M}_{r}(\mathbb{C})$ the $C^{\infty}$ map defined by $\pi_{D}(S)=S D S^{-1}$. With the same name we note its restriction to the unitary group: $\pi_{D}: \mathcal{U}(r) \rightarrow \mathcal{U}(D)$.

Proposition 2.2.2. The similarity orbit $\mathcal{S}(D)$ is a $C^{\infty}$ submanifold of $\mathcal{M}_{r}(\mathbb{C})$, and the projection $\pi_{D}: \mathcal{G} l_{r}(\mathbb{C}) \rightarrow \mathcal{S}(D)$ becomes a submersion. Moreover, $\mathcal{U}(D)$ is a compact submanifold of $\mathcal{S}(D)$, which consists of the normal elements of $\mathcal{S}(D)$, and $\pi_{D}: \mathcal{U}(r) \rightarrow \mathcal{U}(D)$ is a submersion. In particular, the maps $\pi_{D}$ have $C^{\infty}$ (similarity and unitary) local cross sections.

For every $N=U D U^{*} \in \mathcal{U}(D)$, it is well known (and easy to see) that

$$
T_{N} \mathcal{S}(D)=T_{I}\left(\pi_{N}\right)\left(\mathcal{M}_{r}(\mathbb{C})\right)=\left\{[A, N]=A N-N A: A \in \mathcal{M}_{r}(\mathbb{C})\right\} .
$$

In particular

$$
\begin{aligned}
T_{D} \mathcal{S}(D) & =\left\{A D-D A: A \in \mathcal{M}_{r}(\mathbb{C})\right\} \\
& =\left\{X \in \mathcal{M}_{r}(\mathbb{C}): X_{i j}=0 \text { for every }(i, j) \text { such that } d_{i}=d_{j}\right\}
\end{aligned}
$$

Note that,

$$
\begin{aligned}
T_{N} \mathcal{S}(D) & =\left\{[A, N]=A N-N A: A \in \mathcal{M}_{r}(\mathbb{C})\right\} \\
& =\left\{\left(U B U^{*}\right) U D U^{*}-U D U^{*}\left(U B U^{*}\right): B \in \mathcal{M}_{r}(\mathbb{C})\right\} \\
& =\left\{U[B, D] U^{*}=B D-D B: B \in \mathcal{M}_{r}(\mathbb{C})\right\}=U\left(T_{D} \mathcal{S}(D)\right) U^{*}
\end{aligned}
$$

On the other hand, since $T_{I} \mathcal{U}(r)=\mathcal{M}_{r}^{a h}(\mathbb{C})=\left\{A \in \mathcal{M}_{r}(\mathbb{C}): A^{*}=-A\right\}$, we obtain

$$
\begin{aligned}
& T_{D} \mathcal{U}(D)=T_{I}\left(\pi_{D}\right)\left(\mathcal{M}_{r}^{a h}(\mathbb{C})\right)=\left\{[A, D]=A D-D A: A \in \mathcal{M}_{r}^{a h}(\mathbb{C})\right\} \quad \text { and } \\
& T_{N} \mathcal{U}(D)=\left\{[A, N]=A N-N A: A \in \mathcal{M}_{r}^{a h}(\mathbb{C})\right\}=U\left(T_{D} \mathcal{U}(D)\right) U^{*}
\end{aligned}
$$


Finally, along this paper we shall consider on $\mathcal{S}(D)$ (and in $\mathcal{U}(D)$ ) the Riemannian structure inherited from $\mathcal{M}_{r}(\mathbb{C})$ (using the usual inner product on their tangent spaces). For $S, T \in \mathcal{S}(D)$, we denote by $\operatorname{dist}(S, T)$ the Riemannian distance between $S$ and $T$ (in $\mathcal{S}(D)$ ). Observe that, for every $U \in \mathcal{U}(r)$, one has that $U \mathcal{S}(D) U^{*}=\mathcal{S}(D)$ and the map $T \mapsto U T U^{*}$ is isometric, on $\mathcal{S}(D)$, with respect to the Riemannian metric as well as with respect to the $\|\cdot\|_{2}$ metric of $\mathcal{M}_{r}(\mathbb{C})$.

\section{$2.3 \lambda$-Aluthge transforms}

Definition 2.3.1. Let $T \in \mathcal{M}_{r}(\mathbb{C})$, and suppose that $T=U|T|$ is the polar decomposition of $T$. Let $\lambda \in(0,1)$. Then, we define the $\lambda$-Aluthge transform of $T$ in the following way:

$$
\Delta_{\lambda}(T)=|T|^{\lambda} U|T|^{1-\lambda}
$$

We denote by $\Delta_{\lambda}^{n}(T)$ the n-times iterated $\lambda$-Aluthge transform of $T$, i.e.

$$
\Delta_{\lambda}^{0}(T)=T ; \quad \text { and } \quad \Delta_{\lambda}^{n}(T)=\Delta_{\lambda}\left(\Delta_{\lambda}^{n-1}(T)\right) \quad n \in \mathbb{N} .
$$

The following proposition contains some properties of $\lambda$-Aluthge transforms which follows easily from its definition.

Proposition 2.3.2. Let $T \in \mathcal{M}_{r}(\mathbb{C})$ and $\lambda \in(0,1)$. Then:

1. $\Delta_{\lambda}(c T)=c \Delta_{\lambda}(T)$ for every $c \in \mathbb{C}$.

2. $\Delta_{\lambda}\left(V T V^{*}\right)=V \Delta_{\lambda}(T) V^{*}$ for every $V \in \mathcal{U}(r)$.

3. If $T=T_{1} \oplus T_{2}$ then $\Delta_{\lambda}(T)=\Delta_{\lambda}\left(T_{1}\right) \oplus \Delta_{\lambda}\left(T_{2}\right)$.

4. $\left\|\Delta_{\lambda}(T)\right\|_{2} \leqslant\|T\|_{2}$.

5. $T$ and $\Delta_{\lambda}(T)$ have the same characteristic polynomial.

6. In particular, $\sigma\left(\Delta_{\lambda}(T)\right)=\sigma(T)$.

The following theorem states the regularity properties of $\lambda$-Aluthge transforms.

Theorem 2.3.3. The $\lambda$-Aluthge transform is continuous in $\mathcal{M}_{r}(\mathbb{C})$. Moreover, the map $(0,1) \times$ $\mathcal{G} l_{r}(\mathbb{C}) \ni(\lambda, T) \mapsto \Delta_{\lambda}(T)$ is of class $C^{\infty}$.

Proof. The continuity part was proved in [4] (see also [10]). If $T \in \mathcal{G} l_{r}(\mathbb{C})$, then

$$
\Delta_{\lambda}(T)=|T|^{\lambda} T|T|^{-\lambda}, \quad \lambda \in(0,1) .
$$

This clearly implies regularity, since the map

$$
(0,1) \times \mathcal{G} l_{r}(\mathbb{C}) \ni(\lambda, T) \longmapsto|T|^{\lambda}=\left(T^{*} T\right)^{\lambda / 2}=\exp \left(\lambda / 2 \log T^{*} T\right)
$$

is of class $C^{\infty}$.

The following result is proved in [4]: 
Proposition 2.3.4. Given $T \in \mathcal{M}_{r}(\mathbb{C})$ and $\lambda \in(0,1)$, the limit points of the sequence $\left\{\Delta_{\lambda}^{n}(T)\right\}_{n \in \mathbb{N}}$ are normal. Moreover, if $L$ is a limit point, then $\sigma(L)=\sigma(T)$ with the same algebraic multiplicity. In particular, for each $\lambda \in(0,1)$, one has that $\Delta_{\lambda}(T)=T$ if and only if $T$ is normal.

Finally, we mention a result concerning the Jordan structure of Aluthge transforms proved in [4. We need the following definitions.

Definition 2.3.5. Let $T \in \mathcal{M}_{r}(\mathbb{C})$ and $\mu \in \mathbb{C}$. We denote

1. $m(T, \mu)$ the algebraic multiplicity of $\mu$ for $T$, i.e. the maximum $n \in \mathbb{N}$ such that $(x-\mu)^{n}$ divides the characteristic polynomial of $T$.

2. $m_{0}(T, \mu)=\operatorname{dim} \operatorname{ker}(T-\mu I)$, the geometric multiplicity of $\mu$.

Observe that Proposition 2.3 .2 says that $m(T, \mu)=m\left(\Delta_{\lambda}(T), \mu\right)$ for every $\mu \in \mathbb{C}$.

Proposition 2.3.6. Let $T \in \mathcal{M}_{r}(\mathbb{C})$.

1. If $0 \in \sigma(T)$, then, there exists $n \in \mathbb{N}$ such that

$$
m(T, 0)=m_{0}\left(\Delta_{\lambda}^{n}(T), 0\right)=\operatorname{dim} \operatorname{ker}\left(\Delta_{\lambda}^{n}(T)\right)
$$

2. For every $\mu \in \sigma(T), m_{0}(T, \mu) \leqslant m_{0}\left(\Delta_{\lambda}(T), \mu\right)$.

Observe that this implies that, if $T$ is diagonalizable (i.e. $m_{0}(T, \mu)=m(T, \mu)$ for every $\mu$ ), then also $\Delta_{\lambda}(T)$ is diagonalizable.

Remark 2.3.7. Let $T \in \mathcal{G} l_{r}(\mathbb{C})$ with polar decomposition $T=U|T|$. The Duggal or 1-Aluthge transform of $T$ is $\Delta_{1}(T)=|T| U$. It is easy to see that the map $\Delta_{1}: \mathcal{G} l_{r}(\mathbb{C}) \rightarrow \mathcal{G} l_{r}(\mathbb{C})$ is continuous and that $\Delta_{1}(T)=T$ if and only if $T$ is normal. Observe that $U \in \mathcal{U}(r)$, so that $\Delta_{1}(T)=U T U^{*}$, and the distance of $\Delta_{1}(T)$ to the normal matrices is the same as the distance of $T$ to the normal matrices. All these facts imply the iterated Duggal transforms $\Delta_{1}^{n}(T)$ can not converge, unless $T$ is normal.

\section{Convergence}

In this section, we prove the convergence of iterated $\lambda$ - Aluthge transforms for every diagonalizable matrix and $\lambda \in(0,1)$. Throughout the next subsections, a diagonal matrix $D=$ $\operatorname{diag}\left(d_{1}, \ldots, d_{n}\right) \in \mathcal{G} l_{r}(\mathbb{C})$ is fixed. For every $j \in\{1, \ldots, n\}$, let $d_{j}=e^{i \theta_{j}}\left|d_{j}\right|$ be the polar decomposition of $d_{j}$, where $\theta_{j} \in[0,2 \pi]$.

\subsection{Reduction to the invertible case}

We claim that the proof of the convergence of iterated $\lambda$ - Aluthge transforms can be reduced to the invertible case. Indeed, let $T \in \mathcal{M}_{r}(\mathbb{C})$ be a a diagonalizable matrix with polar decomposition $T=U|T|$. As $R(T)$ is a (oblique) complement of $\operatorname{ker} T=\operatorname{ker}|T|^{\lambda}$ and $R\left(U|T|^{1-\lambda}\right)=R(T)$, it holds that

$$
R\left(\Delta_{\lambda}(T)\right)=R\left(|T|^{\lambda} U|T|^{1-\lambda}\right)=R(|T|) .
$$


On the other hand, it is easy to see that $\operatorname{ker} \Delta_{\lambda}(T)=\operatorname{ker}|T|^{1-\lambda}=\operatorname{ker}|T|$, which is orthogonal to $R(|T|)$. By Proposition 2.3.6, after one iteration we get that

$$
\Delta_{\lambda}(T)=\left(\begin{array}{cc}
T_{1} & 0 \\
0 & 0
\end{array}\right) \quad \begin{aligned}
& \operatorname{ker} T^{\perp} \\
& \operatorname{ker} T
\end{aligned}
$$

where $T_{1}$ is invertible and diagonalizable on $\operatorname{ker} T^{\perp}$. By Proposition 2.3.6 again,

$$
\Delta_{\lambda}^{n}(T)=\left(\begin{array}{cc}
\Delta_{\lambda}^{n-1}\left(T_{1}\right) & 0 \\
0 & 0
\end{array}\right) \begin{aligned}
& \operatorname{ker} T^{\perp} \\
& \operatorname{ker} T
\end{aligned} \quad, \quad \text { for every } n \in \mathbb{N} .
$$

Hence, the convergence of $\left\{\Delta_{\lambda}^{n}(T)\right\}_{n \in \mathbb{N}}$ is equivalent to the convergence of $\left\{\Delta_{\lambda}^{n}\left(T_{1}\right)\right\}_{n \in \mathbb{N}}$.

\subsection{Main Theorem}

Reduced the problem to the invertible case, the key tool, which allows to use the stable manifold theorem 2.1.3, is Theorem 3.2.1 below. The proof of this theorem is rather long and technical, for this reason, we postpone it until section 5 , and we continue in this section with its consequences.

Theorem 3.2.1. Let $\lambda \in(0,1)$. The $\lambda$-Aluthge transform $\Delta_{\lambda}(\cdot): \mathcal{S}(D) \rightarrow \mathcal{S}(D)$ is a $C^{\infty}$ map, and for every $N \in \mathcal{U}(D)$, there exists a subspace $\mathcal{E}_{N, \lambda}^{s}$ in the tangent space $T_{N} \mathcal{S}(D)$ such that

1. $T_{N} \mathcal{S}(D)=\mathcal{E}_{N, \lambda}^{s} \oplus T_{N} \mathcal{U}(D)$;

2. Both, $\mathcal{E}_{N, \lambda}^{s}$ and $T_{N} \mathcal{U}(D)$, are $T_{N} \Delta_{\lambda}$-invariant;

3. $\left\|\left.T_{N} \Delta_{\lambda}\right|_{\mathcal{E}_{N, \lambda}^{s}}\right\| \leq k_{D, \lambda}<1$, where

$$
k_{D, \lambda}=\max \left\{\max _{\left|d_{i}\right| \neq\left|d_{j}\right|} \frac{\left|d_{j}\right|^{1-\lambda}\left|d_{i}\right|^{\lambda}+\left|d_{i}\right|^{1-\lambda}\left|d_{j}\right|^{\lambda}}{\left|d_{i}\right|+\left|d_{j}\right|}, \max _{\theta_{i} \neq \theta_{j}}\left|\lambda\left(e^{i\left(\theta_{j}-\theta_{i}\right)}-1\right)+1\right|\right\}<1 .
$$

4. If $U \in \mathcal{U}(r)$ satisfies $N=U D U^{*}$, then $\mathcal{E}_{N, \lambda}^{s}=U\left(\mathcal{E}_{D, \lambda}^{s}\right) U^{*}$.

In particular, the map $\mathcal{U}(D) \ni N \mapsto \mathcal{E}_{N, \lambda}^{s}$ is smooth. This fact can be formulated in terms of the projections $P_{N, \lambda}$ onto $\mathcal{E}_{N, \lambda}^{s}$ parallel to $T_{N} \mathcal{U}(D), N \in \mathcal{U}(D)$.

Proof. See Section 5

Corollary 3.2.2. Let $D=\operatorname{diag}\left(d_{1}, \ldots, d_{r}\right) \in \mathcal{M}_{r}(\mathbb{C})$ be an invertible diagonal matrix and $\lambda \in(0,1)$. Let $\mathcal{E}_{N, \lambda}^{s}$ and $k_{D}$ as in Theorem 3.2.1. Then, in $\mathcal{S}(D)$ there exists a $\Delta_{\lambda}$-invariant $C^{\infty}$-pre-lamination $\left\{\mathcal{W}_{N, \lambda}\right\}_{N \in \mathcal{U}(D)}$ of class $C^{\infty}$ such that, for every $N \in \mathcal{U}(D)$,

1. $\mathcal{W}_{N, \lambda}$ is a $C^{\infty}$ submanifold of $\mathcal{S}(D)$.

2. $T_{N} \mathcal{W}_{N, \lambda}=\mathcal{E}_{N, \lambda}^{s}$.

3. If $k_{D}<\rho<1$, then $\operatorname{dist}\left(\Delta_{\lambda}^{n}(T)-N\right) \leq \operatorname{dist}(T, N) \rho^{n}$, for every $T \in \mathcal{W}_{N, \lambda}$.

4. If $N_{1} \neq N_{2}$ then $\mathcal{W}_{N_{1}, \lambda} \cap \mathcal{W}_{N_{2}, \lambda}=\varnothing$.

5. There exists an open subset $\mathcal{W}(D)$ of $\mathcal{S}(D)$ such that 
a. $\mathcal{U}(D) \subseteq \mathcal{W}(D) \subseteq \bigcup_{N \in \mathcal{U}(D)} \mathcal{W}_{N, \lambda}$, and

b. The map $p: \mathcal{W}(D) \rightarrow \mathcal{U}(D)$, defined by $p(T)=N$ if $T \in \mathcal{W}_{N, \lambda}$, is of class $C^{\infty}$.

Proof. By Theorem 3.2.1, for every $k_{D}<\rho<1, \mathcal{U}(D)$ is $\rho$-pseudo hyperbolic for $\Delta_{\lambda}$ (see Definition 2.1.2), and it consists of fixed points. Thus, by Theorem 2.1.3, we get a $C^{\infty}$ and $\Delta_{\lambda}$-invariant pre-lamination of class $C^{\infty},\left\{\mathcal{W}_{N, \lambda}\right\}_{N \in \mathcal{U}(D)}$ which satisfies all the properties of our statement.

\subsection{Convergence for fixed $\lambda$.}

Using the previous results, we can apply exactly the same techniques as in our previous work [5], and to obtain for every $\lambda \in(0,1)$ the same results about $\Delta_{\lambda}(\cdot)$ as those obtained for the classical Aluthge transform $\Delta_{1 / 2}(\cdot)$. We state these properties in the following Theorem. The basic idea is to apply Proposition 2.3.4 in order to assure that the iterations go into the open set $\mathcal{W}(D)$, where the smooth projection $p$ can be used. Although the proof of this theorem is omitted to avoid repetitions, Proposition 4.1 .2 below gives a detailed proof.

Theorem 3.3.1. Let $\lambda \in(0,1)$ :

1. Given a diagonalizable matrix $T \in \mathcal{M}_{r}(\mathbb{C})$, the sequence $\left\{\Delta_{\lambda}^{n}(T)\right\}_{n \in \mathbb{N}}$ converges and its limit will be denoted by $\Delta_{\lambda}^{\infty}(T)$.

2. Let $D \in \mathcal{M}_{r}(\mathbb{C})$ be diagonal. Then the sequence $\left\{\Delta_{\lambda}^{n}\right\}_{n \in \mathbb{N}}$, restricted to the similarity orbit $\mathcal{S}(D)$, converges uniformly on compact sets to the map $\Delta_{\lambda}^{\infty}: \mathcal{S}(D) \rightarrow \mathcal{U}(D)$, which is of class $C^{\infty}$. In particular, $\Delta_{\lambda}^{\infty}$ is a $C^{\infty}$ retraction from $\mathcal{S}(D)$ onto $\mathcal{U}(D)$.

Remark 3.3.2. Let $D \in \mathcal{M}_{r}(\mathbb{C})$ be diagonal. For every $N \in \mathcal{U}(D)$ and $\lambda \in(0,1)$, denote

$$
\mathcal{W}_{N, \lambda}^{+}=\left\{T \in \mathcal{S}(D): \Delta_{\lambda}^{\infty}(T)=N\right\} .
$$

Since $T \mapsto \Delta_{\lambda}^{\infty}(T)$ is a $C^{\infty}$ retraction from $\mathcal{S}(D)$ onto $\mathcal{U}(D)$, each $\mathcal{W}_{N, \lambda}^{+}$is a $C^{\infty}$ submanifold of $\mathcal{S}(D)$; and $\mathcal{S}(D)$ stands as the (disjoint) union of these sheets. On the other hand, the submanifolds $\mathcal{W}_{N, \lambda}^{+}$are prolongations of the sheets $\mathcal{W}_{N, \lambda}$ of Corollary 3.2.2. Indeed, for every $N \in \mathcal{U}(D)$ and $\lambda \in(0,1)$,

$$
\mathcal{W}_{N, \lambda} \subseteq \mathcal{W}_{N, \lambda}^{+} \quad \text { and } \quad \mathcal{W}(D) \cap \mathcal{W}_{N, \lambda}=\mathcal{W}(D) \cap \mathcal{W}_{N, \lambda}^{+},
$$

by Corollary 3.2.2. Then $T_{N} \mathcal{W}_{N, \lambda}^{+}=T_{N} \mathcal{W}_{N, \lambda}=\mathcal{E}_{N, \lambda}^{s}$.

\section{Regularity properties of $\Delta_{\lambda}^{\infty}(T)$}

As in section 3 , we fix $D=\operatorname{diag}\left(d_{1}, \ldots, d_{r}\right) \in \mathcal{G} l_{r}(\mathbb{C})$. Observe that, using the continuity of $\Delta_{\lambda}(\cdot)$ with respect to $\lambda$ (Thm. 2.3.3), and the fact that the convergence of its iterations to the limit map $\Delta_{\lambda}^{\infty}(\cdot)$ is uniform on compact subsets of $\mathcal{S}(D)$ (for each $\lambda$ ), one can show that the map

$$
(0,1) \times \mathcal{S}(D) \ni(\lambda, T) \longmapsto \Delta_{\lambda}^{\infty}(T)
$$

is continuous. The purpose of this section is to prove that this map is smooth. Firstly we shall analyze the invertible case. Then, in subsection 4.2 we shall see that the invertibility hypothesis on $D$ can be dropped. 


\subsection{On the orbit $\mathcal{S}(D)$.}

Denote by $\mathcal{S}_{L}(D)=(0,1) \times \mathcal{S}(D)$ and $\mathcal{U}_{L}(D)=(0,1) \times \mathcal{U}(D)$. Consider the map

$$
\Delta: \mathcal{S}_{L}(D) \rightarrow \mathcal{S}_{L}(D) \text { given by } \Delta(\lambda, T)=\left(\lambda, \Delta_{\lambda}(T)\right), \quad(\lambda, T) \in \mathcal{S}_{L}(D) .
$$

Remark 4.1.1. Using Theorems 2.3.3, 3.2.1 and 3.3.1 and Proposition 2.3.4, one can deduce easily the following properties:

1. $\mathcal{S}_{L}(D)$ is a $C^{\infty}$ manifold, and $\mathcal{U}_{L}(D)$ is a submanifold of $\mathcal{S}_{L}(D)$.

2. The map $\Delta$ is of class $C^{\infty}$.

3. The submanifold $\mathcal{U}_{L}(D)$ coincides with the set of all fixed points of $\Delta$.

4. For every $(\lambda, T) \in \mathcal{S}_{L}(D)$, it holds that

$$
\Delta^{n}(\lambda, T)=\left(\lambda, \Delta_{\lambda}^{n}(T)\right) \underset{n \rightarrow \infty}{\longrightarrow}\left(\lambda, \Delta_{\lambda}^{\infty}(T)\right) .
$$

5. For every $N \in \mathcal{U}(D)$ and $\lambda \in(0,1)$, one can describe the tangent spaces as

$$
\begin{gathered}
T_{(\lambda, N)} \mathcal{U}_{L}(D)=T_{N} \mathcal{U}(D) \oplus \mathbb{R}, \quad \text { and } \\
T_{(\lambda, N)} \mathcal{S}_{L}(D)=T_{N} \mathcal{S}(D) \oplus \mathbb{R}=\mathcal{E}_{N, \lambda}^{s} \oplus T_{N} \mathcal{U}(D) \oplus \mathbb{R}=\mathcal{E}_{N, \lambda}^{s} \oplus T_{(\lambda, N)} \mathcal{U}_{L}(D)
\end{gathered}
$$

where the spaces $\mathcal{E}_{N, \lambda}^{s}$ are those of Theorem 3.2 .1 .

6. By Remark 5.2.2, the projections $Q_{N, \lambda}=P_{N, \lambda}+P_{\mathbb{R}}$ onto $\mathcal{E}_{N, \lambda}^{s}$ parallel to $T_{(\lambda, N)} \mathcal{U}_{L}(D)$, given by the above decomposition, satisfy that the map

$$
(0,1) \times \mathcal{U}(D) \ni(\lambda, N) \longmapsto Q_{N, \lambda}
$$

is of class $C^{\infty}$.

Proposition 4.1.2. The iterates $\Delta^{n}$ of the map $\Delta: \mathcal{S}_{L}(D) \rightarrow \mathcal{S}_{L}(D)$ defined in Eq. (4.1) satisfy

$$
\Delta^{n}(\lambda, T) \underset{n \rightarrow \infty}{\longrightarrow} \Delta^{\infty}(\lambda, T) \quad \text { for every } \quad(\lambda, T) \in \mathcal{S}_{L}(D)
$$

where $\Delta^{\infty}: \mathcal{S}_{L}(D) \rightarrow \mathcal{U}_{L}(D)$ is a $C^{\infty}$ retraction.

Proof. Fix $\left(\lambda_{0}, T_{0}\right) \in \mathcal{S}_{L}(D)$. Let $0<\lambda_{1}<\lambda_{0}<\lambda_{2}<1$, and consider the submaniflods

$$
\mathcal{S}_{o}(D)=\left(\lambda_{1}, \lambda_{2}\right) \times \mathcal{S}(D) \subseteq \mathcal{S}_{L}(D) \quad \text { and } \quad \mathcal{U}_{o}(D)=\left(\lambda_{1}, \lambda_{2}\right) \times \mathcal{U}(D) .
$$

Observe that $\mathcal{S}_{O}(D)$ is open in $\mathcal{S}_{L}(D)$, it is $\Delta$ invariant, and its fixed points coincide with $\mathcal{U}_{o}(D)$, which is also open in $\mathcal{U}_{L}(D)$. Fix $\rho \in(0,1)$ such that the constants (of Theorem 3.2.1) $k_{D, \lambda}<\rho$ for every $\lambda \in\left(\lambda_{1}, \lambda_{2}\right)$. Observe that, for every $(\lambda, N) \in \mathcal{U}_{o}(D)$,

$$
\left.T_{(\lambda, N)} \Delta\right|_{\mathcal{E}_{N, \lambda}^{s}}=\left.T_{N} \Delta_{\lambda}\right|_{\mathcal{E}_{N, \lambda}^{s}} \subseteq \mathcal{E}_{N, \lambda}^{s} \subseteq T_{(\lambda, N)} \mathcal{S}_{L}(D)
$$

Using this fact, and items 5 and 6 of Remark 4.1.1, one can assure that $\mathcal{U}_{o}(D)$ is $\rho$-pseudo hyperbolic for $\Delta: \mathcal{S}_{o}(D) \rightarrow \mathcal{S}_{o}(D)$ (see Definition 2.1.2) consisting of fixed points. Thus, by Theorem 2.1.3, we get a $C^{\infty}$ and $\Delta$-invariant pre-lamination $\left\{\mathcal{W}_{(\lambda, N)}\right\}_{(\lambda, N) \in \mathcal{U}_{o}(D)}$ of class $C^{\infty}$ such that, for every $(\lambda, N) \in \mathcal{U}_{o}(D)$, 
1. $\mathcal{W}_{(\lambda, N)}$ is a $C^{\infty}$ submanifold of $\mathcal{S}_{o}(D)$.

2. $T_{(\lambda, N)} \mathcal{W}_{(\lambda, N)}=\mathcal{E}_{(\lambda, N)}^{s}$.

3. $\operatorname{dist}\left(\Delta^{n}(\mu, T)-(\lambda, N)\right) \leq \rho^{n} \operatorname{dist}((\mu, T),(\lambda, N))$, for every $(\mu, T) \in \mathcal{W}_{(\lambda, N)}$. Observe that this implies that,

$$
\text { if }(\mu, T) \in \mathcal{W}_{(\lambda, N)} \text {, then } \mu=\lambda \text {. }
$$

4. If $\left(\mu_{1}, N_{1}\right) \neq\left(\mu_{2}, N_{2}\right)$ then $\mathcal{W}_{\left(\mu_{1}, N_{1}\right)} \cap \mathcal{W}_{\left(\mu_{2}, N_{2}\right)}=\varnothing$.

5. There exists an open subset $\mathcal{W}(D)$ of $\mathcal{S}_{o}(D)$ such that

a. $\mathcal{U}_{o}(D) \subseteq \mathcal{W}(D) \subseteq \bigcup_{(\lambda, N) \in \mathcal{U}(D)} \mathcal{W}_{(\lambda, N)}$.

b. The map $p: \mathcal{W}(D) \rightarrow \mathcal{U}_{o}(D)$, defined by

$$
p(\lambda, T)=(\lambda, N) \quad \text { if } \quad(\lambda, T) \in \mathcal{W}_{(\lambda, N)},
$$

is well defined and of class $C^{\infty}$.

By item 2 of Remark 4.1.1, the map $\Delta$ and its iterations are $C^{\infty}$ functions. Hence, there exist an open set $\mathcal{U} \subseteq \mathcal{S}_{o}(D)$ and $k \in \mathbb{N}$ such that $\left(\lambda_{0}, T_{0}\right) \in \mathcal{U}$ and $\Delta^{k}(\mathcal{U}) \subseteq \mathcal{W}(D)$. By the regularity the projection $p$, one can deduce that the map $\mathcal{S}_{o}(D) \ni(\lambda, T) \longmapsto p\left(\Delta^{k}(\lambda, T)\right)$ is of class $C^{\infty}$ on $\mathcal{U}$. Now, if $\Delta^{k}(\lambda, T) \in \mathcal{W}_{(\lambda, N)}$ then

$$
p\left(\Delta^{k}(\lambda, T)\right)=N=\lim _{n \rightarrow \infty} \Delta^{n}(\lambda, T),
$$

by item 3 . Hence $\Delta^{\infty}$ is well defined and of class $C^{\infty}$.

Theorem 4.1.3. Let $D \in \mathcal{D}_{r}(\mathbb{C})$ be invertible. Then the map

$$
\Delta_{\infty}:(0,1) \times \mathcal{S}(D) \rightarrow \mathcal{U}(D) \quad \text { given by } \quad \Delta_{\infty}(\lambda, T)=\Delta_{\lambda}^{\infty}(T), \quad(\lambda, T) \in \mathcal{S}_{L}(D)
$$

is of class $C^{\infty}$.

Proof. Let $\Delta^{\infty}: \mathcal{S}_{L}(D) \rightarrow \mathcal{U}_{L}(D)$ be the $C^{\infty}$ retraction of Proposition 4.1.2, By Eq. (4.2),

$$
\Delta^{\infty}(\lambda, T)=\left(\lambda, \Delta_{\infty}(\lambda, T)\right) \quad \text { for every } \quad(\lambda, T) \in \mathcal{S}_{o}(D) .
$$

This implies that also the map $\Delta_{\infty}$ is of class $C^{\infty}$.

Remark 4.1.4. Observe that Proposition 4.1.2 and Eq. (4.2), before taking the limit, also show Theorem 3.3.1, whose proof was omitted. On the other hand, they also shows that the map $\Delta^{\infty}: \mathcal{S}_{L}(D) \rightarrow \mathcal{U}_{L}(D)$ given by $\Delta^{\infty}(\lambda, T)=\left(\lambda, \Delta_{\lambda}^{\infty}(T)\right)$ is a $C^{\infty}$ retraction from $\mathcal{S}_{L}(D)$ onto $\mathcal{U}_{L}(D)$. Therefore, for every $(\lambda, N) \in \mathcal{U}_{L}(D)$, the set $\mathcal{W}_{(\lambda, N)}^{+}=\left(\Delta^{\infty}\right)^{-1}(\lambda, N)$ is a smooth submanifold of $\mathcal{S}_{L}(D)$. Observe that, by Remark 3.3.2. Eq. (4.2) and (4.3), we have that $\mathcal{W}_{(\lambda, N)}^{+}=\{\lambda\} \times \mathcal{W}_{\lambda, N}^{+}$and

$$
\mathcal{W}_{(\lambda, N)}=\mathcal{W}(D) \cap \mathcal{W}_{(\lambda, N)}^{+}=\mathcal{W}(D) \cap\left(\{\lambda\} \times \mathcal{W}_{\lambda, N}^{+}\right)=\{\lambda\} \times \mathcal{W}_{\lambda, N}
$$

where $\mathcal{W}_{\lambda, N}^{+}$are the sheets described in Remark 3.3.2, and $\mathcal{W}_{\lambda, N}$ are those sheets which appear in Corollary 3.2.2, This can be described as the fact that "the submanifolds $\mathcal{W}_{\lambda, N}$ move smoothly with $\lambda "$. 


\subsection{The not invertible case}

As in section 3 , all the results of this section still hold if the diagonal matrix $D \in \mathcal{M}_{r}(\mathbb{C})$ is not invertible. Indeed, suppose that rk D $=s<r$, and fix $T \in \mathcal{S}(D)$ and $\lambda \in(0,1)$. By Eq. (3.1),

$$
\Delta_{\lambda}(T)=\left(\begin{array}{cc}
T_{1} & 0 \\
0 & 0
\end{array}\right) \quad \begin{aligned}
& \operatorname{ker} T^{\perp} \\
& \operatorname{ker} T
\end{aligned}
$$

where $T_{1}$ is invertible and diagonalizable on $\operatorname{ker} T^{\perp}$. The same happens for every matrix $S \in \mathcal{S}(D)$. Denote by $P=P_{\operatorname{ker} T}$ and $\mathcal{U}(P)=\left\{U P U^{*}: U \in \mathcal{U}(r)\right\}$ its unitary orbit. Consider the map

$$
Q: \mathcal{S}(D) \rightarrow \mathcal{U}(P) \quad \text { given by } \quad Q(S)=P_{\text {ker } S}=P_{\text {ker } \Delta_{\lambda}(S)}, \quad S \in \mathcal{S}(D) .
$$

This map takes values in $\mathcal{U}(P)$ because rk $\mathrm{Q}(\mathrm{S})=\operatorname{rk} \mathrm{P}=s$ for every $S \in \mathcal{S}(D)$. Also, $Q$ is of class $C^{\infty}$, since $S \mapsto \Delta_{\lambda}(S)$ is smooth, and $\Delta_{\lambda}(S) \mapsto P_{\text {ker } \Delta_{\lambda}(S)}$ is polynomial. By Proposition 2.2.2, there exist an open set $\mathcal{W} \subseteq \mathcal{U}(P)$ which contains $P$, and a $C^{\infty}$ local cross section $\sigma: \mathcal{W} \rightarrow \mathcal{U}(r)$, in the sense that $\sigma(R) P \sigma(R)^{*}=R$ for every $R \in \mathcal{W}$. Let $\mathcal{V} \subseteq \mathcal{S}(D)$ be an open set such that $T \in \mathcal{V}$ and $Q(\mathcal{V}) \subseteq \mathcal{W}$. Denote by $\eta=\sigma \circ Q: \mathcal{V} \rightarrow \mathcal{U}(r)$. Then $\eta$ is also of class $C^{\infty}$. So, for every $\lambda \in(0,1)$ and $S \in \mathcal{V}$, there exists $\gamma(S, \lambda) \in L\left(\operatorname{ker} T^{\perp}\right)$ such that

$$
\eta(S)^{*} \Delta_{\lambda}(S) \eta(S)=\left(\begin{array}{cc}
\gamma(S, \lambda) & 0 \\
0 & 0
\end{array}\right) \begin{aligned}
& \operatorname{ker} T^{\perp} \\
& \operatorname{ker} T
\end{aligned} \text { and } \gamma(S, \lambda) \in \mathcal{S}\left(T_{1}\right) \subseteq L\left(\operatorname{ker} T^{\perp}\right)
$$

Therefore, using that $\Delta_{\lambda}\left(U T U^{*}\right)=U \Delta_{\lambda}(T) U^{*}$ for every $U \in \mathcal{U}(r)$, we obtain

$$
\Delta_{\lambda}^{\infty}(S)=A d_{\eta(S)} \circ\left(\begin{array}{cc}
\Delta_{\lambda}^{\infty}(\gamma(S, \lambda)) & 0 \\
0 & 0
\end{array}\right) \begin{aligned}
& \operatorname{ker} T^{\perp} \\
& \operatorname{ker} T
\end{aligned} \quad, \quad \text { for every } S \in \mathcal{W} .
$$

Then the regularity of $(\lambda, S) \mapsto \Delta_{\lambda}^{\infty}(S)$ can be deduced from the regularity of the maps $(0,1) \times$ $\mathcal{V} \ni(\lambda, S) \mapsto \gamma(S, \lambda)$ and $(0,1) \times \mathcal{S}\left(T_{1}\right) \ni(\lambda, A) \mapsto \Delta_{\lambda}^{\infty}(A)$. Hence, the reduction to the invertible case is proved.

\subsection{Different eigenvalues.}

Let $\mathcal{D}_{r}^{*}(\mathbb{C})$ be the set of diagonalizable and invertible matrices in $\mathcal{M}_{r}(\mathbb{C})$ with $r$ different eigenvalues (i.e. every eigenvalue has algebraic multiplicity equal to one). Observe that $\mathcal{D}_{r}^{*}(\mathbb{C})$ is an open dense subset of $\mathcal{M}_{r}(\mathbb{C})$ and it is invariant by the Aluthge transform.

Theorem 4.3.1. The map $\Delta_{\infty}:(0,1) \times \mathcal{D}_{r}^{*}(\mathbb{C}) \rightarrow \mathcal{U}(D)$ given by

$$
\Delta_{\infty}(\lambda, T)=\Delta_{\lambda}^{\infty}(T), \quad(\lambda, T) \in(0,1) \times \mathcal{D}_{r}^{*}(\mathbb{C})
$$

is of class $C^{\infty}$.

Proof. It follows from a straightforward combination of the techniques of section 3.2 of [5] and those of the previous sections. We omit the details. 


\subsection{The map $\lambda \mapsto \Delta_{\lambda}^{\infty}(T)$ for fixed $T$.}

Definition 4.4.1. Let $T \in \mathcal{M}_{r}(\mathbb{C})$ be a diagonalizable. We call $R_{T}:(0,1) \rightarrow \mathcal{M}_{r}(\mathbb{C})$ the map given by $R_{T}(\lambda)=\Delta_{\lambda}^{\infty}(T)$, for $\lambda \in(0,1)$.

The following question arises naturally: Is the map $R_{T}$ constant for every diagonalizable matrix $T \in \mathcal{M}_{r}(\mathbb{C})$ ?. Numerical examples shows that the question has a negative answer in general. Indeed, taking the $2 \times 2$ matrix $T=\left(\begin{array}{rr}3 & 0 \\ -2 & 1\end{array}\right)$, numerical computations show that

$$
R_{T}(0.3) \cong\left(\begin{array}{cc}
2.2273 & 0.97380 \\
0.97380 & 1.7726
\end{array}\right) \text { and } R_{T}(0.7) \cong\left(\begin{array}{cc}
1.37162 & -0.77790 \\
-0.77790 & 2.62838
\end{array}\right)
$$

Nevertheless, it was proved in [4, Thm. 4.9] that, if $D=\operatorname{diag}\left(d_{1}, d_{2}\right) \in \mathcal{M}_{2}(\mathbb{C})$ and $\left|d_{1}\right|=\left|d_{2}\right|$, then $R_{T}$ is constant for every $T \in \mathcal{S}(D)$. Our next result shows that, for a diagonal matrix $D=\operatorname{diag}\left(d_{1}, \ldots, d_{r}\right) \in \mathcal{M}_{r}(\mathbb{C})$, this may happen only if $\left|d_{1}\right|=\ldots=\left|d_{r}\right|$.

Proposition 4.4.2. Let $D \in \mathcal{M}_{r}(\mathbb{C})$ be diagonal and invertible. If $D$ has two eigenvalues with distinct moduli, then there exists $T \in \mathcal{S}(D)$ such that the map $R_{T}$ is not constant.

The proof of Proposition 4.4.2 follows directly from the next two Lemmas. But let us make first some comments about this problem:

Remark 4.4.3. Despite Proposition 4.4.2, given $D=\operatorname{diag}\left(d_{1}, \ldots, d_{r}\right) \in \mathcal{M}_{r}(\mathbb{C})$ with $\left|d_{i}\right| \neq\left|d_{j}\right|$ for some $i, j \in\{1, \ldots, r\}$, the similarity orbit $\mathcal{S}(D)$ may contain (non normal) matrices $T$ for which the map $R_{T}$ is constant. In fact, consider the following example: let $D=\operatorname{diag}(1,-1,2) \in$ $\mathcal{M}_{3}(\mathbb{C})$, take $D_{1}=\operatorname{diag}(1,-1), T_{1} \in \mathcal{S}\left(D_{1}\right)$ and $T=\left(\begin{array}{cc}T_{1} & 0 \\ 0 & 2\end{array}\right) \in \mathcal{S}(D)$, then

$$
R_{T}(\lambda)=\Delta_{\lambda}^{\infty}(T)=\left(\begin{array}{cc}
\Delta_{\lambda}^{\infty}\left(T_{1}\right) & 0 \\
0 & 2
\end{array}\right)=\left(\begin{array}{cc}
R_{T_{1}}(\lambda) & 0 \\
0 & 2
\end{array}\right), \text { for every } \lambda \in(0,1)
$$

But the map $R_{T_{1}}$ is constant by [4, Thm 4.9] (see also Proposition 4.4 .6 below). Observe that this example includes several cases where $T \notin \mathcal{U}(D)$ (otherwise, the map would be trivially constant). On the other hand, Proposition 4.4 .2 is not longer true if $D$ is not invertible. This fact can be immediately tested by taking $D$ with $\sigma(D)=\{0,1\}$ (i.e., if $D$ is a projection).

Lemma 4.4.4. Let $D \in \mathcal{M}_{r}(\mathbb{C})$ be diagonal and invertible. If the map $R_{T}$ is constant for every $T \in \mathcal{S}(D)$, then the distribution of subspaces

$$
(0,1) \ni \lambda \longmapsto \mathcal{E}_{N, \lambda}^{s}
$$

given by Theorem 3.2.1 must be constant for every $N \in \mathcal{U}(D)$.

Proof. Using the notations of Remark 3.3 .2 and Corollary 3.2.2, each submanifold $\mathcal{W}_{N, \lambda}^{+}$consist of those matrices $T$ such that $R_{T}(\lambda)=\Delta_{\lambda}^{\infty}(T)=N$. But if all the maps $\Delta_{\lambda}^{\infty}(\cdot)$ are the same on $\mathcal{S}(D)$, then the submanifolds $\mathcal{W}_{N, \lambda}^{+}$must agree for different lambdas. Finally observe that, by Remark 3.3 .2 and Corollary $3.2 .2, \mathcal{E}_{N, \lambda}^{s}$ is the tangent space of $\mathcal{W}_{N, \lambda}^{+}$at $N$, for every $N \in \mathcal{U}(D)$ and every $\lambda \in(0,1)$.

Lemma 4.4.5. Let $D=\operatorname{diag}\left(d_{1}, \ldots, d_{n}\right) \in \mathcal{M}_{r}(\mathbb{C})$ be diagonal and invertible. Then the following conditions are equivalent: 
1. The distribution of subspaces $(0,1) \ni \lambda \longmapsto \mathcal{E}_{N, \lambda}^{s}$ given by Theorem 3.2.1 is constant for every $N \in \mathcal{U}(D)$.

2. $\left|d_{i}\right|=\left|d_{j}\right|$ for every $1 \leq i, j \leq r$.

Moreover, in this case, $\mathcal{E}_{N, \lambda}^{s}=\left[T_{D} \mathcal{U}(D)\right]^{\perp}$ for every $\lambda \in(0,1)$.

Proof. Since the proof uses several results and notation from section 5, we postpone it until that section. See Remark 5.2.1.

Now, the natural question is, what happens if $D$ has all its eigenvalues of the same modulus? We first study a particular case:

Proposition 4.4.6. Let $D \in \mathcal{M}_{r}(\mathbb{C})$ be diagonal such that $\sigma(D)=\left\{d_{1}, d_{2}\right\}$ with $\left|d_{1}\right|=\left|d_{2}\right|$. Then the map $R_{T}$ is constant for every $T \in \mathcal{S}(D)$.

Proof. Let $T \in \mathcal{S}(D)$. Denote $\mathcal{S}_{i}=\operatorname{ker}\left(T-d_{i} I\right)$ for $i=1,2$, and by $Q$ the oblique projection onto $\mathcal{S}_{1}$ given by the decomposition $\mathbb{C}^{r}=\mathcal{S}_{1} \oplus \mathcal{S}_{2}$. Then $T=d_{1} Q+d_{2}(I-Q)$. Assume that $k=\operatorname{dim} \mathcal{S}_{2} \geq n=\operatorname{dim} \mathcal{S}_{1}$. In matrix terms, there exists $A \in L\left(\mathcal{S}_{1}^{\perp}, \mathcal{S}_{1}\right)$ such that

$$
Q=\left(\begin{array}{cc}
I & A \\
0 & 0
\end{array}\right) \mathcal{S}_{1}^{\perp} \quad \text { 列 } \quad T=\left(\begin{array}{cc}
d_{1} I & \left(d_{1}-d_{2}\right) A \\
0 & d_{2} I
\end{array}\right) \mathcal{S}_{1}^{\perp}
$$

We can assume that $\mathcal{S}_{1}=\left\{x \in \mathbb{C}^{r}: x_{i}=0\right.$ for $\left.i>n\right\}$, by a unitary conjugation, which commutes with $\Delta_{\lambda}^{\infty}$. In this case, $A \in \mathcal{M}_{n, k}(\mathbb{C})$. By the decomposition in singular values of $B=\left(d_{1}-d_{2}\right) A$, there exist $U \in \mathcal{U}(n)$ and $V \in \mathcal{U}(k)$ such that $U B V^{*}=\Sigma(B)$, where $\Sigma(B)=\left(\Sigma_{0}(B), 0\right) \in \mathcal{M}_{n, k}(\mathbb{R})$ with $\Sigma_{0}(B)=\operatorname{diag}\left(s_{1}(B), \ldots, s_{n}(B)\right) \in \mathcal{M}_{n}(\mathbb{R})$, and we add a $n \times(k-n)$ block of zeros on the right. If $W=U \oplus V \in \mathcal{U}(r)$, then

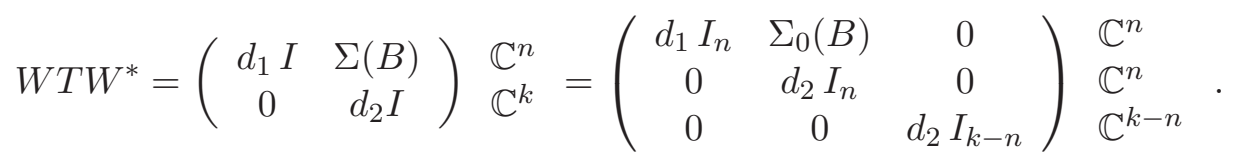

Moreover, there exists a permutation matrix $S \in \mathcal{U}(r)$ which rearranges the entries of $W T W^{*}$ in such a way that

$$
M=S W T W^{*} S^{*}=\bigoplus_{i=1}^{n}\left(\begin{array}{cc}
d_{1} & s_{i}(B) \\
0 & d_{2}
\end{array}\right) \oplus d_{2} I_{k-n} .
$$

As before, it suffices to show that the map $R_{M}$ is constant. But now Proposition 2.3.6 assures that

$$
R_{M}(\lambda)=\Delta_{\lambda}^{\infty}(M)=\bigoplus_{i=1}^{n} \Delta_{\lambda}^{\infty}\left(\begin{array}{cc}
d_{1} & s_{i}(B) \\
0 & d_{2}
\end{array}\right) \oplus d_{2} I_{k-n} \quad \text { for every } \lambda \in(0,1)
$$

Finally, it was proved in [4, Thm. 4.9] that each map $\lambda \mapsto \Delta_{\lambda}^{\infty}\left(\begin{array}{cc}d_{1} & s_{i}(B) \\ 0 & d_{2}\end{array}\right)$ is constant. 
Remark 4.4.7. The case $D=D^{*}$ in Proposition 4.4.6, is particularly interesting, because in this case all iterated $\lambda$-Alutge transforms (and the limit) can be explicitly computed for every $E \in \mathcal{S}(D)$, even in the infinite dimensional case: Let $\mathcal{H}$ be a Hilbert space and $D \in L_{s a}(\mathcal{H})$ such that $\sigma(D)=\{1,-1\}$. Observe that if $E \in \mathcal{S}(D)$, then $E^{2}=E$. The geometry of $\mathcal{S}(D)$ has been widely studied (see [17], 6] and [7]). Given $E \in \mathcal{S}(D)$, observe that, if $L=|E|$, then $\left|E^{*}\right|=L^{-1}$. Therefore, if $E=R L$ is the polar decomposition of $E$, with $R \in \mathcal{U}(r)$, then $R L=E=E^{-1}=L^{-1} R^{*}$. But the right polar decomposition is $E=\left|E^{*}\right| R$ (with the same $R$ ), so that $R=R^{-1}=R^{*}$ is a unitary reflection and $R L=L^{-1} R$. Moreover, $R \in \mathcal{U}(D)$, because $E=R L=L^{-1 / 2} R L^{1 / 2}$.

The map $E \mapsto R$ is the retraction $p: \mathcal{S}(D) \rightarrow \mathcal{U}(D)$ deeply studied in [18. We claim that $p=\Delta_{1 / 2}(\cdot)=\Delta_{\lambda}^{\infty}(\cdot)$ for every $\lambda \in(0,1)$. Indeed, observe that

$$
\Delta_{1 / 2}(E)=L^{1 / 2} R L^{1 / 2}=L^{1 / 2} L^{-1 / 2} R=R
$$

and, for every $\lambda \in(0,1), \Delta_{\lambda}(E)=L^{\lambda} R L^{1-\lambda}=R L^{1-2 \lambda}$, so that $\Delta_{\lambda}^{2}(E)=R L^{(1-2 \lambda)^{2}}$, since $\Delta_{\lambda}(E)=R L^{1-2 \lambda}$ is the polar decomposition of $\Delta_{\lambda}(E)$. Inductively, for every $n \in \mathbb{N}$,

$$
\Delta_{\lambda}^{n}(E)=R L^{(1-2 \lambda)^{n}} \underset{n \rightarrow \infty}{\longrightarrow} R=\Delta_{\lambda}^{\infty}(E),
$$

since $|1-2 \lambda|<1$. Then the map $\lambda \mapsto R_{E}(\lambda)=\Delta_{\lambda}^{\infty}(E)$ is constant, but the rate of convergence is very different for each $\lambda$, being slower when $\lambda$ tends to 0 or 1 . On the other hand, the sheets $\mathcal{W}_{R, \lambda}^{+}$of Remark 3.3 .2 can be characterized as

$$
\mathcal{W}_{R, \lambda}^{+}=\left\{R M: M \in \mathcal{G} l(\mathcal{H})^{+} \quad \text { and } \quad R M=M^{-1} R\right\} .
$$

The geometry of these hyperbolic manifolds is also deeply studied in [7].

If $D$ has all its eigenvalues of the same modulus and $\sigma(D)$ has more than two elements, we do not have an answer to the above question, but we have made several computational experiments. In all the tested examples, the map $R_{T}$ fails to be constant for some $T$ in the orbit, even if $D$ satisfies some algebraic condition such as $D^{3}=I$. This suggests the following conjecture:

Conjecture. Let $D \in \mathcal{M}_{r}(\mathbb{C})$ be diagonal and invertible. Then the map $R_{T}$ is constant for every $T \in \mathcal{S}(D)$ if and only if $\sigma(D)=\left\{d_{1}, d_{2}\right\}$ with $\left|d_{1}\right|=\left|d_{2}\right|$.

Remark 4.4.8. Observe that it would be sufficient to consider the $3 \times 3$ case, because we can use a similar reduction to the one used in Remark 4.4.3.

Example 4.4.9. Let $a, b, c \in \mathbb{R}^{+}$such that $a b c=1$, and let $T$ be the $3 \times 3$ matrix defined by

$$
T=\left(\begin{array}{lll}
0 & 0 & 1 \\
1 & 0 & 0 \\
0 & 1 & 0
\end{array}\right) \cdot\left(\begin{array}{lll}
a & 0 & 0 \\
0 & b & 0 \\
0 & 0 & c
\end{array}\right)=U|T|
$$

Computing its spectrum, one shows that $T \in \mathcal{S}(U)$. On the other hand, since $U$ is a permutation matrix, for every diagonal matrix $D \in \mathcal{M}_{3}(\mathbb{C})$ both $U D U^{*}$ and $U^{*} D U$ are also diagonal matrices. In particular, $\left|T^{*}\right|=U|T| U^{*}$ is diagonal and commutes with $|T|$. Let $\lambda \in(0,1)$. Then

$$
\Delta_{\lambda}(T)=|T|^{\lambda} U|T|^{1-\lambda}=U\left(U^{*}\left(|T|^{\lambda}\left|T^{*}\right|^{1-\lambda}\right) U\right)=U\left|\Delta_{\lambda}(T)\right|,
$$


where the last equality holds by the uniqueness of the polar decomposition. Note that $\left|\Delta_{\lambda}(T)\right|$ is diagonal with det $\left|\Delta_{\lambda}(T)\right|=1$. An inductive argument shows that $\Delta_{\lambda}^{n}(T)=U D_{n}$ for every $n \in \mathbb{N}$, where each $D_{n}$ is a positive diagonal matrix. As $T \in \mathcal{S}(U)$, then $\Delta_{\lambda}^{\infty}(T) \in \mathcal{U}(U) \subseteq \mathcal{U}(r)$. Hence $D_{n} \underset{n \rightarrow \infty}{\longrightarrow} I$ and $\Delta_{\lambda}^{\infty}(T)=U$. The same happens for any $\lambda \in(0,1)$, therefore, the function $R_{T}(\lambda)$ is constant. This example does not contradicts the Conjecture, because all the matrices studied satisfy that $|T|$ and $\left|T^{*}\right|$ commute, so that they are not dense in $\mathcal{S}(U)$.

\section{The proof of Theorem 3.2.1.}

As in Section 3, in this section we fix an invertible diagonal matrix $D \in \mathcal{M}_{r}(\mathbb{C})$ whose diagonal entries are denoted by $\left(d_{1}, \ldots, d_{n}\right)$. For every $j \in\{1, \ldots, n\}$, let $d_{j}=e^{i \theta_{j}}\left|d_{j}\right|$ be the polar decomposition of $d_{j}$, where $\theta_{j} \in[0,2 \pi]$.

\subsection{Matricial characterization of $T_{N} \Delta_{\lambda}$}

Definition 5.1.1. Given $A, B \in \mathcal{M}_{r}(\mathbb{C}), A \circ B$ denotes their Hadamard product, that is, if $A=\left(A_{i j}\right)$ and $B=\left(B_{i j}\right)$, then $(A \circ B)_{i j}=A_{i j} B_{i j}$. With respect to this product, each matrix $A \in \mathcal{M}_{r}(\mathbb{C})$ induces an operator $\Psi_{A}$ on $\mathcal{M}_{r}(\mathbb{C})$ defined by $\Psi_{A}(B)=A \circ B, B \in \mathcal{M}_{r}(\mathbb{C})$.

Remark 5.1.2. In what follows, we shall state several definitions and results taken from Section 4 of our previous work [5]:

1. By Eq. (2.3), the tangent space $T_{D} \mathcal{S}(D)$ consists on those matrices $X \in \mathcal{M}_{r}(\mathbb{C})$ such that $X_{i j}=0$ if $d_{i}=d_{j}$. Then $T_{D} \mathcal{S}(D)$ reduces the operator $\Psi_{A}$, for every $A \in \mathcal{M}_{r}(\mathbb{C})$. This is the reason why, from now on, we shall consider all these operators as acting on $T_{D} \mathcal{S}(D)$. Restricted in this way, it holds that

$$
\left\|\Psi_{A}\right\|=\sup \left\{\|A \circ B\|_{2}: B \in T_{D} \mathcal{S}(D) \text { and }\|B\|_{2}=1\right\}=\max _{d_{i} \neq d_{j}}\left|A_{i j}\right|,
$$

since $\Psi_{A}$ is a diagonal operator on the Hilbert space $\left(T_{D} \mathcal{S}(D),\|\cdot\|_{2}\right)$.

2. Let $P_{\mathbb{R e}}$ and $P_{\mathbb{I} m}$ be the projections defined on $T_{D} \mathcal{S}(D)$ by

$$
P_{\mathbb{R} e}(B)=\frac{B+B^{*}}{2} \quad \text { and } \quad P_{\mathbb{I m}}(B)=\frac{B-B^{*}}{2} \quad, \quad B \in T_{D} \mathcal{S}(D) .
$$

That is, $P_{\mathbb{R e}}$ (resp. $P_{\mathbb{I} m}$ ) is the restriction to $T_{D} \mathcal{S}(D)$ of the orthogonal projection onto the subspace of hermitian (resp. anti-hermitian) matrices.

3. Observe that, for every $K \in \mathcal{M}_{r}^{a h}(\mathbb{C})$ (i.e., such that $K^{*}=-K$ ) and $B \in \mathcal{M}_{r}(\mathbb{C})$ it holds that

$$
K \circ P_{\mathbb{R e}}(B)=P_{\mathbb{I m}}(K \circ B) \quad \text { and } \quad K \circ P_{\mathbb{I m}}(B)=P_{\mathbb{R e}}(K \circ B) .
$$

4. Denote by $Q_{D}$ the orthogonal projection from $T_{D} \mathcal{S}(D)$ onto $\left(T_{D} \mathcal{U}(D)\right)^{\perp}$.

5. Let $J, K \in \mathcal{M}_{r}(\mathbb{C})$ be the matrices defined by

$$
K_{i j}=\left\{\begin{array}{ll}
\left|d_{j}-d_{i}\right| \operatorname{sgn}(j-i) & \text { if } d_{i} \neq d_{j} \\
0 & \text { if } d_{i}=d_{j}
\end{array}\right. \text { and }
$$




$$
J_{i j}= \begin{cases}\left(d_{j}-d_{i}\right) K_{i j}^{-1} & \text { if } d_{i} \neq d_{j} \\ 1 & \text { if } d_{i}=d_{j}\end{cases}
$$

for $1 \leq i, j \leq r$. Then

(a) For every $A \in \mathcal{M}_{r}(\mathbb{C}), A D-D A=J \circ K \circ A$.

(b) It holds that $Q_{D}=\Psi_{J} P_{\mathbb{I m}} \Psi_{J}^{-1}$.

(c) If $H \in \mathcal{M}_{r}^{h}(\mathbb{C})$ (i.e., if $H^{*}=H$ ), then $Q_{D} \Psi_{H}=\Psi_{H} Q_{D}$.

6. Let $\lambda \in(0,1)$ and $N \in \mathcal{U}(D)$ and let $Q_{N}$ be the orthogonal projection from $T_{N} \mathcal{S}(D)$ onto $\left(T_{N} \mathcal{U}(D)\right)^{\perp}$. Then $T_{N} \Delta_{\lambda}$ has the following $2 \times 2$ matrix decomposition

$$
T_{N} \Delta_{\lambda}=\left(\begin{array}{ll}
A_{1 N}(\lambda) & 0 \\
A_{2 N}(\lambda) & I
\end{array}\right) \quad \begin{array}{r}
Q_{N} \\
I-Q_{N}
\end{array}
$$

because $T_{N} \Delta_{\lambda}$ acts as the identity on $T_{N} \mathcal{U}(D)$.

7. Let $A \in \mathcal{M}_{r}(\mathbb{C})$ and let $\gamma: \mathbb{R} \rightarrow L(\mathcal{H})^{+}$be the curve defined by

$$
\gamma(t)=\left(e^{t A} D e^{-t A}\right)^{*}\left(e^{t A} D e^{-t A}\right)=e^{-t A^{*}} D^{*} e^{t A^{*}} e^{t A} D e^{-t A}
$$

If $R, T^{+}$and $T^{-} \in \mathcal{M}_{r}(\mathbb{C})$ are defined by $R_{i j}=2 \bar{d}_{i} d_{j}, T_{i j}^{+}=\left|d_{i}\right|^{2}+\left|d_{j}\right|^{2}$ and $T_{i j}^{-}=$ $\left|d_{j}\right|^{2}-\left|d_{i}\right|^{2}, i, j \in \mathbb{I}_{r}$, then

$$
\gamma^{\prime}(0)=\left(R-T^{+}\right) \circ P_{\mathbb{R} e}(A)+T^{-} \circ P_{\mathbb{I m}}(A) .
$$

The following classical result, proved by Dalekiüi and Kreln in 1951 ([8] and [9]) will be useful in the sequel (see also the book [12]).

Theorem 5.1.3. Let $I, J \subseteq \mathbb{R}$ be open intervals and let $\gamma: I \rightarrow \mathcal{M}_{r}^{h}(\mathbb{C})$ be a $C^{1}$ curve such that $\sigma(\gamma(t)) \subseteq J$ for every $t \in I$. Let $f: J \rightarrow \mathbb{R}$ be a $C^{1}$ map. Suppose that $\gamma\left(t_{0}\right)=\operatorname{diag}\left(a_{1}, \ldots, a_{r}\right)$ for some $t_{0} \in I$. Then

$$
(f \circ \gamma)^{\prime}\left(t_{0}\right)=M_{f} \circ \gamma^{\prime}\left(t_{0}\right)
$$

where $M_{f} \in \mathcal{M}_{n}(\mathbb{R})$ is defined by

$$
\left(M_{f}\right)_{i j}=\left\{\begin{array}{ll}
\frac{f\left(a_{j}\right)-f\left(a_{i}\right)}{a_{j}-a_{i}} & \text { if } a_{i} \neq a_{j} \\
f^{\prime}\left(a_{i}\right) & \text { if } a_{i}=a_{j}
\end{array}, \quad \text { for } \quad i, j \in \mathbb{I}_{r} .\right.
$$

Corollary 5.1.4. Let $A$ and $\gamma$ be as in 7 of Remark 5.1.2, and let $\lambda \in(0,1)$. Then $\left(\gamma^{\lambda / 2}\right)^{\prime}(0)=$ $M_{\lambda / 2} \circ \gamma^{\prime}(0)$, where $M_{\lambda / 2} \in \mathcal{M}_{r}(\mathbb{C})$ is the matrix given by

$$
\left(M_{\lambda / 2}\right)_{i j}=\left\{\begin{array}{ll}
\frac{\left|d_{j}\right|^{\lambda}-\left|d_{i}\right|^{\lambda}}{\left|d_{j}\right|^{2}-\left|d_{i}\right|^{2}} & \text { if }\left|d_{i}\right| \neq\left|d_{j}\right| \\
\frac{\lambda}{2}\left|d_{i}\right|^{\lambda-2} & \text { if }\left|d_{i}\right|=\left|d_{j}\right|
\end{array} \quad, \quad i, j \in \mathbb{I}_{r} .\right.
$$


Proof. Apply Theorem 5.1 .3 to $f(t)=t^{\frac{\lambda}{2}}$. Use that $\gamma(0)=\operatorname{diag}\left(\left|d_{1}\right|^{2}, \ldots,\left|d_{r}\right|^{2}\right)$.

Recall that $Q_{D}$ denotes the orthogonal projection from $T_{D} \mathcal{S}(D)$ onto $\left(T_{D} \mathcal{U}(D)\right)^{\perp}$.

Proposition 5.1.5. Fix $\lambda \in(0,1)$. Then there exists a matrix $H(\lambda) \in \mathcal{M}_{r}(\mathbb{C})$ such that $Q_{D}\left(T_{D} \Delta_{\lambda}\right) Q_{D}=Q_{D} \Psi_{P_{\mathbb{R e}} H(\lambda)} Q_{D}$ and

$$
\left(I-Q_{D}\right)\left(T_{D} \Delta_{\lambda}\right) Q_{D}=\left(I-Q_{D}\right) \Psi_{P_{\mathbb{I m}} H(\lambda)} Q_{D}
$$

The entries of $H_{1}(\lambda)=P_{\mathbb{R e}} H(\lambda)$ are the following: for every $1 \leq i, j \leq r$, denote by $A_{i j}=$ $\left|d_{j}\right|^{2-\lambda}\left|d_{i}\right|^{\lambda}-\left|d_{i}\right|^{2-\lambda}\left|d_{j}\right|^{\lambda}$ and $B_{i j}=\left|d_{j}\right|^{1+\lambda}\left|d_{i}\right|^{1-\lambda}-\left|d_{i}\right|^{1+\lambda}\left|d_{j}\right|^{1-\lambda}$. Then

$$
H_{1}(\lambda)_{i j}=\left\{\begin{array}{ll}
\frac{A_{i j}+e^{i\left(\theta_{j}-\theta_{i}\right)} B_{i j}}{\left|d_{j}\right|^{2}-\left|d_{i}\right|^{2}} & \text { if }\left|d_{i}\right| \neq\left|d_{j}\right| \\
\lambda\left(e^{i\left(\theta_{j}-\theta_{i}\right)}-1\right)+1 & \text { if }\left|d_{i}\right|=\left|d_{j}\right|
\end{array} .\right.
$$

The proof of this proposition follows the same steps as the proof of of [5, Prop. 4.1.5], but using now Corollary [5.1.4, and items 5 and 7 of Remark 5.1.2. For this reason we shall give only and sketched version, pointed out the main differences and the technical difficulties that appear when we loose the symmetry of the case $\lambda=1 / 2$.

Sketch of proof. Fix $X=A D-D A \in T_{D} \mathcal{S}(D)$, for some $A \in \mathcal{M}_{r}(\mathbb{C})$. Then

$$
T_{D} \Delta_{\lambda}(X)=\left.\frac{d}{d t} \Delta_{\lambda}\left(e^{t A} D e^{-t A}\right)\right|_{t=0} .
$$

Let $\gamma(t)=\left(e^{t A} D e^{-t A}\right)^{*}\left(e^{t A} D e^{-t A}\right)=e^{-t A^{*}} D^{*} e^{t A^{*}} e^{t A} D e^{-t A}$. In terms of $\gamma$, we can write the curve $\Delta_{\lambda}\left(e^{t A} D e^{-t A}\right)$ in the following way

$$
\Delta_{\lambda}\left(e^{t A} D e^{-t A}\right)=\gamma^{\lambda / 2}(t)\left(e^{t A} D e^{-t A}\right) \gamma^{-\lambda / 2}(t) .
$$

Since $\gamma^{\lambda / 2} \gamma^{-\lambda / 2}=I$, then $\left(\gamma^{-\lambda / 2}\right)^{\prime}(0)=-\gamma^{-\lambda / 2}(0)\left(\gamma^{\lambda / 2}\right)^{\prime}(0) \gamma^{-\lambda / 2}(0)$. Using this identity, easy computations show that

$$
T_{D} \Delta_{\lambda}(X)=\left(\left(\gamma^{\lambda / 2}\right)^{\prime}(0) D-D\left(\gamma^{\lambda / 2}\right)^{\prime}(0)\right)|D|^{-\lambda}+|D|^{\lambda}(A D-D A)|D|^{-\lambda} .
$$

If we define the matrices $L, N \in \mathcal{M}_{r}(\mathbb{C})$ by $N_{i j}=\left|d_{j}\right|^{-\lambda}$ and $L_{i j}=\left|d_{i}\right|^{\lambda}\left|d_{j}\right|^{-\lambda}$ and take $J, K \in \mathcal{M}_{r}(\mathbb{C})$ as in 5 of Remark 5.1 .2 . Then

$$
T_{D} \Delta_{\lambda}(X)=N \circ\left(J \circ K \circ\left(\gamma^{\lambda / 2}\right)^{\prime}(0)\right)+L \circ(J \circ K \circ A) .
$$

Using Corollary 5.1.4 and 7 of Remark 5.1.2, we get

$$
\begin{aligned}
T_{D} \Delta_{\lambda}(A D-D A) & =N \circ J \circ K \circ M_{\lambda / 2} \circ\left[\left(R-T^{+}\right) \circ P_{\mathbb{R e}}(A)+T^{-} \circ P_{\mathbb{I m}}(A)\right] \\
& +L \circ J \circ K \circ A .
\end{aligned}
$$

where $R, T^{+}$and $T^{-}$are the matrices defined in 7 of Remark 5.1.2, and $M_{\lambda / 2}$ is the matrix defined in Eq. (5.4) of Corollary 5.1.4. 
Now, we shall express $T_{D} \Delta_{\lambda}(A D-D A)$ in terms of $A D-D A=J \circ K \circ A$. Following the same steps as in Proposition 4.1.5 of [5], we arrive to the formula

$$
T_{D} \Delta_{\lambda}(A D-D A)=\left(\Psi_{H(\lambda)} Q_{D}+\left(I-Q_{D}\right)\right)(A D-D A),
$$

where $H(\lambda)=M_{\lambda / 2} \circ N \circ\left(R-T^{+}\right)+L$. Given $X \in R\left(Q_{D}\right)$, then $\Psi_{J}^{-1}(X) \in \mathcal{M}_{r}^{a h}(\mathbb{C})$ and

$$
\begin{aligned}
Q_{D}\left(T_{D} \Delta_{\lambda}\right) Q_{D}(X) & =Q_{D}(H(\lambda) \circ X)=\left(\Psi_{J} P_{\mathbb{I m}} \Psi_{J}^{-1}\right)(H(\lambda) \circ X) \\
& =J \circ\left(P_{\mathbb{I m}}\left(H(\lambda) \circ \Psi_{J}^{-1} X\right)\right) \\
& =\frac{1}{2} J \circ\left(H(\lambda) \circ \Psi_{J}^{-1}(X)+H(\lambda)^{*} \circ \Psi_{J}^{-1}(X)\right) \\
& =J \circ P_{\mathbb{R} e} H(\lambda) \circ \Psi_{J}^{-1}(X)=P_{\mathbb{R e} e} H(\lambda) \circ X=Q_{D} \Psi_{P_{\mathbb{R} e} H(\lambda)}(X) .
\end{aligned}
$$

Analogously, one shows that $\left(I-Q_{D}\right)\left(T_{D} \Delta\right) Q_{D}(X)=\left(I-Q_{D}\right) \Psi_{P_{\mathbb{I m}} H(\lambda)}(X)$. In order to prove Eq. (5.5), recall that $H(\lambda)=M_{\lambda / 2} \circ N \circ\left(R-T^{+}\right)+L$. Hence, $H(\lambda)_{i j}=\left(M_{\lambda / 2}\right)_{i j}\left(\left|d_{j}\right|^{-\lambda}\left(2 \bar{d}_{i} d_{j}-\right.\right.$ $\left.\left.\left(\left|d_{i}\right|^{2}+\left|d_{j}\right|^{2}\right)\right)\right)+\left|d_{i}\right|^{\lambda}\left|d_{j}\right|^{-\lambda}$. Suppose that $\left|d_{i}\right| \neq\left|d_{j}\right|$. Straightforward computations, using Corollary 5.1.4, show that

$$
\begin{aligned}
P_{\mathbb{R e}} H(\lambda)_{i j}= & \frac{1}{2}\left(\frac{2\left|d_{j}\right|^{2-\lambda}\left|d_{i}\right|^{\lambda}+2 \bar{d}_{i} d_{j}-2 \bar{d}_{i} d_{j}\left|d_{i}\right|^{\lambda}\left|d_{j}\right|^{-\lambda}-\left|d_{i}\right|^{2}-\left|d_{j}\right|^{2}}{\left|d_{j}\right|^{2}-\left|d_{i}\right|^{2}}\right)+ \\
& \frac{1}{2}\left(\frac{2\left|d_{i}\right|^{2-\lambda}\left|d_{j}\right|^{\lambda}+2 \bar{d}_{i} d_{j}-2 \bar{d}_{i} d_{j}\left|d_{j}\right|^{\lambda}\left|d_{i}\right|^{-\lambda}-\left|d_{j}\right|^{2}-\left|d_{i}\right|^{2}}{\left|d_{i}\right|^{2}-\left|d_{j}\right|^{2}}\right) \\
= & \frac{A_{i j}+e^{i\left(\theta_{j}-\theta_{i}\right)} B_{i j}}{\left|d_{j}\right|^{2}-\left|d_{i}\right|^{2}}
\end{aligned}
$$

where $A_{i j}$ and $B_{i j}$ are those of the statement. If $\left|d_{i}\right|=\left|d_{j}\right|$, then

$$
H(\lambda)_{i j}=\frac{\lambda}{2}\left|d_{i}\right|^{\lambda-2}\left(\left|d_{i}\right|^{2-\lambda} 2\left(e^{i\left(\theta_{j}-\theta_{i}\right)}-1\right)\right)+1=\lambda\left(e^{i\left(\theta_{j}-\theta_{i}\right)}-1\right)+1 .
$$

So that $H(\lambda)_{i j}=\overline{H(\lambda)_{j i}}=P_{\mathbb{R e}} H(\lambda)_{i j}=H_{1}(\lambda)_{i j}=\lambda\left(e^{i\left(\theta_{j}-\theta_{i}\right)}-1\right)+1$.

Remark 5.1.6. Using the notations of Proposition 5.1.5, let $H_{2}(\lambda)=P_{\mathbb{I} m} H(\lambda)$.

1. If $\left|d_{i}\right|=\left|d_{j}\right|$, as we observed at the end of the proof of Proposition 5.1.5, $H(\lambda)_{i j}=\overline{H(\lambda)_{j i}}$. Hence, $H_{2}(\lambda)_{i j}=0$.

2. If $\left|d_{i}\right| \neq\left|d_{j}\right|$, denote $a=\frac{\left|d_{j}\right|}{\left|d_{i}\right|} \neq 1$ and $\alpha=e^{i\left(\theta_{j}-\theta_{i}\right)}$. Then one can show that

$$
H_{2}(\lambda)_{i j}=\left(a-a^{-1}\right)^{-1}\left[a^{1-\lambda}+a^{\lambda-1}+\alpha\left(2-a^{1-\lambda}-a^{\lambda-1}\right)-a-a^{-1}\right]
$$

while $H_{1}(\lambda)_{i j}=\left(a-a^{-1}\right)^{-1}\left[a^{1-\lambda}-a^{\lambda-1}+\alpha\left(a^{\lambda}-a^{-\lambda}\right)\right]$. 
Corollary 5.1.7. Given $N \in \mathcal{U}(D)$, consider the matrix decomposition

$$
T_{N} \Delta_{\lambda}=\left(\begin{array}{ll}
A_{1 N}(\lambda) & 0 \\
A_{2 N}(\lambda) & I
\end{array}\right) \quad \begin{aligned}
& Q_{N} \\
& I-Q_{N}
\end{aligned}
$$

as in Eq. (5.2). Then $\left\|A_{1 N}(\lambda)\right\| \leq k_{D, \lambda}$, where

$$
k_{D, \lambda}=\max \left\{\max _{\left|d_{i}\right| \neq\left|d_{j}\right|} \frac{\left|d_{j}\right|^{1-\lambda}\left|d_{i}\right|^{\lambda}+\left|d_{i}\right|^{1-\lambda}\left|d_{j}\right|^{\lambda}}{\left|d_{i}\right|+\left|d_{j}\right|}, \max _{\theta_{i} \neq \theta_{j}}\left|\lambda e^{i\left(\theta_{j}-\theta_{i}\right)}+1-\lambda\right|\right\}<1 .
$$

Proof. As in the proof of Corollary 4.1 .6 of [5], it holds that $\left\|A_{1 N}(\lambda)\right\|=\left\|A_{1 D}(\lambda)\right\|$ for every $N \in \mathcal{U}(D)$. On the other hand, by Proposition 5.1.5 and its notations, we get that

$$
\left\|A_{1 D}(\lambda)\right\| \leq\left\|\Psi_{H_{1}(\lambda)}\right\|=\max _{d_{i} \neq d_{j}}\left|H_{1}(\lambda)_{i j}\right| .
$$

If $\left|d_{i}\right| \neq\left|d_{j}\right|$, denote $a=\frac{\left|d_{j}\right|}{\left|d_{i}\right|} \neq 1$. As in Proposition [5.1.5, we denote

$$
\begin{gathered}
A_{i j}=\left|d_{j}\right|^{2-\lambda}\left|d_{i}\right|^{\lambda}-\left|d_{i}\right|^{2-\lambda}\left|d_{j}\right|^{\lambda}=\left|d_{j}\right|\left|d_{i}\right|\left(a^{1-\lambda}-a^{\lambda-1}\right) \text { and } \\
B_{i j}=\left|d_{j}\right|^{1+\lambda}\left|d_{i}\right|^{1-\lambda}-\left|d_{i}\right|^{1+\lambda}\left|d_{j}\right|^{1-\lambda}=\left|d_{j}\right|\left|d_{i}\right|\left(a^{\lambda}-a^{-\lambda}\right) .
\end{gathered}
$$

Observe that $A_{i j}$ and $B_{i j}$ have the same sign. So $\left|A_{i j}+B_{i j}\right|= \pm\left(A_{i j}+B_{i j}\right)$ and

$$
\begin{aligned}
\left|H_{1}(\lambda)_{i j}\right|=\left|\frac{A_{i j}-e^{i\left(\theta_{j}-\theta_{i}\right)} B_{i j}}{\left|d_{j}\right|^{2}-\left|d_{i}\right|^{2}}\right| & \leq\left|\frac{A_{i j}+B_{i j}}{\left|d_{j}\right|^{2}-\left|d_{i}\right|^{2}}\right| \\
& =\frac{\left|d_{j}\right|-\left|d_{i}\right|}{\left|d_{j}\right|^{2}-\left|d_{i}\right|^{2}}\left(\left|d_{j}\right|^{1-\lambda}\left|d_{i}\right|^{\lambda}+\left|d_{i}\right|^{1-\lambda}\left|d_{j}\right|^{\lambda}\right) \\
& =\frac{\left|d_{j}\right|^{1-\lambda}\left|d_{i}\right|^{\lambda}+\left|d_{i}\right|^{1-\lambda}\left|d_{j}\right|^{\lambda}}{\left|d_{j}\right|+\left|d_{i}\right|} .
\end{aligned}
$$

This quantity is strictly lower that one (as observed in [13]) because

$$
\left(\left|d_{j}\right|+\left|d_{i}\right|\right)-\left(\left|d_{j}\right|^{1-\lambda}\left|d_{i}\right|^{\lambda}+\left|d_{i}\right|^{1-\lambda}\left|d_{j}\right|^{\lambda}\right)=\left(\left|d_{j}\right|^{\lambda}-\left|d_{i}\right|^{\lambda}\right)\left(\left|d_{j}\right|^{1-\lambda}-\left|d_{i}\right|^{1-\lambda}\right)>0 .
$$

On the other hand, if $\left|d_{i}\right|=\left|d_{j}\right|$ but $\theta_{i} \neq \theta_{j}$, using the triangle inequality we obtain that

$$
\left|H_{1}(\lambda)_{i j}\right|=\left|\lambda\left(e^{i\left(\theta_{j}-\theta_{i}\right)}-1\right)+1\right|=\left|\lambda e^{i\left(\theta_{j}-\theta_{i}\right)}+(1-\lambda)\right|<1 .
$$

In consequence, the bound for $\left\|A_{1 N}(\lambda)\right\|$ is proved.

\subsection{The proof}

Now, we shall restate and prove Theorem 3.2 .1

Theorem. The $\lambda$-Aluthge transform $\Delta_{\lambda}(\cdot): \mathcal{S}(D) \rightarrow \mathcal{S}(D)$ is a $C^{\infty}$ map, and for every $N \in \mathcal{U}(D)$, there exists a subspace $\mathcal{E}_{N, \lambda}^{s}$ in the tangent space $T_{N} \mathcal{S}(D)$ such that

1. $T_{N} \mathcal{S}(D)=\mathcal{E}_{N, \lambda}^{s} \oplus T_{N} \mathcal{U}(D)$; 
2. Both, $\mathcal{E}_{N, \lambda}^{s}$ and $T_{N} \mathcal{U}(D)$, are $T_{N} \Delta_{\lambda}$-invariant;

3. $\left\|\left.T_{N} \Delta_{\lambda}\right|_{\mathcal{E}_{N, \lambda}^{s}}\right\| \leq k_{D, \lambda}<1$, where $k_{D, \lambda}$ is the constant of Corollary 5.1.7

4. If $U \in \mathcal{U}(r)$ satisfies $N=U D U^{*}$, then $\mathcal{E}_{N, \lambda}^{s}=U\left(\mathcal{E}_{D, \lambda}^{s}\right) U^{*}$.

In particular, the map $\mathcal{U}(D) \ni N \mapsto \mathcal{E}_{N, \lambda}^{s}$ is smooth. This fact can be formulated in terms of the projections $P_{N, \lambda}$ onto $\mathcal{E}_{N, \lambda}^{s}$ parallel to $T_{N} \mathcal{U}(D), N \in \mathcal{U}(D)$.

The proof of this theorem follows exactly the same steps as the proof of Theorem 3.1.1 of [5], but now using Corollary [5.1.7, item 5] of Remark [5.1.2, and Proposition 5.1.5] of this work. For this reason, we shall only give a sketch of the proof.

Sketch of proof. Fix $N=U D U^{*} \in \mathcal{U}(D)$. By the statement and the notations of Corollary 5.1.7. $\left\|A_{1 N}(\lambda)\right\|<1$. So the operator $I-A_{1 N}(\lambda)$ acting on $R\left(Q_{N}\right)$ is invertible. Let $\mathcal{E}_{N, \lambda}^{s}$ be the subspace defined by

$$
\mathcal{E}_{N, \lambda}^{s}=\left\{\left(\begin{array}{c}
y \\
-A_{2 N}(\lambda)\left(I-A_{1 N}(\lambda)\right)^{-1} y
\end{array}\right): y \in R\left(Q_{N}\right)\right\}
$$

Now, following the same steps as in the proof of Theorem 3.1.1 of [5] (with minor changes in order to adapts it to our case) we can see that the following properties hold:

- The operator $P_{N, \lambda} \in L\left(T_{N} \mathcal{S}(D)\right)$ given by the matrix

$$
P_{N, \lambda}=\left(\begin{array}{cc}
I & 0 \\
-A_{2 N}(\lambda)\left(I-A_{1 N}(\lambda)\right)^{-1} & 0
\end{array}\right) \quad \begin{gathered}
Q_{N} \\
I-Q_{N}
\end{gathered}
$$

is the projection onto $\mathcal{E}_{N, \lambda}^{s}$ parallel to $T_{N} \mathcal{U}(D)$. Therefore we have the identity $T_{N} \mathcal{U}(D)=$ $\mathcal{E}_{N, \lambda}^{s} \oplus T_{N} \mathcal{U}(D)$.

- Since $T_{N} \Delta_{\lambda}=A d_{U}\left(T_{D} \Delta_{\lambda}\right) A d_{U}^{-1}$, then

$$
P_{N, \lambda}=A d_{U}\left(P_{D, \lambda}\right) A d_{U}^{-1} \quad \text { and } \quad P_{N, \lambda}\left(T_{N} \Delta_{\lambda}\right)=\left(T_{N} \Delta_{\lambda}\right) P_{N, \lambda} .
$$

- $\mathcal{E}_{N, \lambda}^{s}=U\left(\mathcal{E}_{D, \lambda}^{s}\right) U^{*}$ and both, $\mathcal{E}_{N, \lambda}^{s}$ and $T_{N} \mathcal{U}(D)$, are invariant for $T_{N} \Delta_{\lambda}$.

- $\left\|\left.\left(T_{N} \Delta_{\lambda}\right)\right|_{\mathcal{E}_{N, \lambda}^{s}}\right\|=\left\|\left.\left(T_{D} \Delta_{\lambda}\right)\right|_{\mathcal{E}_{D, \lambda}^{s}}\right\|$. So it suffices to show item 3 for $N=D$.

- Let $Y=\left(\begin{array}{c}y \\ -A_{2 D}(\lambda)\left(I-A_{1 D}(\lambda)\right)^{-1} y\end{array}\right) \in \mathcal{E}_{D, \lambda}^{s}$, for some $y \in R\left(Q_{D}\right)$. Then

$$
\begin{aligned}
\left\|\left(T_{D} \Delta_{\lambda}\right) Y\right\|_{2}^{2} & =\left\|A_{1 D}(\lambda) y\right\|_{2}^{2}+\left\|A_{2 D}(\lambda) y-A_{2 D}(\lambda)\left(I-A_{1 D}(\lambda)\right)^{-1} y\right\|_{2}^{2} \\
& \leq k_{D, \lambda}^{2}\|y\|_{2}^{2}+\left\|-A_{2 D}(\lambda) A_{1 D}(\lambda)\left(I-A_{1 D}(\lambda)\right)^{-1} y\right\|_{2}^{2} .
\end{aligned}
$$

where the inequality holds because $\left\|A_{1 D}(\lambda)\right\| \leq k_{D, \lambda}$, by Corollary 5.1.7.

- By item 5 of Remark 5.1.2 and Proposition 5.1.5, we obtain that

$$
\left\|-A_{2 D}(\lambda) A_{1 D}(\lambda)\left(I-A_{1 D}(\lambda)\right)^{-1} y\right\|_{2}^{2} \leq k_{D, \lambda}^{2}\left\|-A_{2 D}(\lambda)\left(I-A_{1 D}(\lambda)\right)^{-1} y\right\|_{2}^{2} .
$$


- $\left\|\left(T_{D} \Delta_{\lambda}\right) Y\right\|_{2}^{2} \leq k_{D, \lambda}^{2}\|y\|_{2}^{2}+k_{D, \lambda}^{2}\left\|-A_{2 D}(\lambda)\left(I-A_{1 D}(\lambda)\right)^{-1} y\right\|_{2}^{2}=k_{D, \lambda}^{2}\|Y\|_{2}^{2}$. Therefore we have that $\left.\left(T_{N} \Delta_{\lambda}\right)\right|_{\mathcal{E}_{N, \lambda}^{s}}$ has norm lower or equal to $k_{D, \lambda}$.

The smoothness of the map $\mathcal{U}(D) \ni N \mapsto \mathcal{E}_{N, \lambda}^{s}$ follows from the existence of $C^{\infty}$ local cross sections for the map $\pi_{D}: \mathcal{U}(r) \rightarrow \mathcal{U}(D)$, stated in Proposition 2.2.2, For example, if $\sigma_{D}: \mathcal{V} \rightarrow$ $\mathcal{U}(r)$ is such a section near $D$, then by Eq. (5.7),

$$
P_{N, \lambda}=A d_{\sigma_{D}(N)} P_{D, \lambda} A d_{\sigma_{D}(N)^{*}} \quad, \quad N \in \mathcal{V} .
$$

This completes the proof.

Remark 5.2.1. Using the notations and the proof of the Theorem, one can see that the significative parts of the projections $P_{N, \lambda}$ are

$$
\begin{aligned}
\left(I-Q_{N}\right) P_{N, \lambda} Q_{N} & =-A_{2 N}(\lambda)\left(I-A_{1 N}(\lambda)\right)^{-1} \\
& =-\left(I-Q_{N}\right) T_{N} \Delta_{\lambda} Q_{N}\left(Q_{N}-Q_{N} T_{N} \Delta_{\lambda} Q_{N}\right)^{-1} .
\end{aligned}
$$

When $N=D$, by 5 of Remark 5.1.2 and Proposition 5.1.5, and using that the matrices $\{1-$ $\left.H_{1}(\lambda)_{i j}\right\}_{i j}$ and $\left\{\left(1-H_{1}(\lambda)_{i j}\right)^{-1}\right\}_{i j}$ are selfadjoint, we have that

$$
\begin{aligned}
\left(I-Q_{D}\right) P_{D, \lambda} Q_{D} & =-\left(I-Q_{D}\right) \Psi_{H_{2}(\lambda)} Q_{D}\left(Q_{D}-Q_{D} \Psi_{H_{1}(\lambda)} Q_{D}\right)^{-1} \\
& =-\left(I-Q_{D}\right) \Psi_{H_{2}(\lambda)}\left(I-\Psi_{H_{1}(\lambda)}\right)^{-1} Q_{D} \\
& =\left(I-Q_{D}\right) \Psi_{G(\lambda)} Q_{D},
\end{aligned}
$$

where $G(\lambda) \in \mathcal{M}_{r}^{a h}(\mathbb{C})$ has entries $G(\lambda)_{i j}=-H_{2}(\lambda)_{i j}\left(1-H_{1}(\lambda)_{i j}\right)^{-1}$. Now, using Remark 5.1.6. we have the following properties, which have been announced in Proposition 4.4.5.

1. If $\left|d_{i}\right|=\left|d_{j}\right|$, then $G(\lambda)_{i j}=0$.

2. Suppose that all the eigenvalues of $D$ have the same moduli. Then, using Eq. (5.6) and Eq. (5.7), we get that $P_{N, \lambda}=Q_{N}$ (i.e., $\left.\mathcal{E}_{N, \lambda}^{s}=\left[T_{N} \mathcal{U}(D)\right]^{\perp}\right)$ for every $\lambda \in(0,1)$ and every $N \in \mathcal{U}(D)$.

3. If $\left|d_{i}\right| \neq\left|d_{j}\right|, a=\frac{\left|d_{j}\right|}{\left|d_{i}\right|} \neq 1$ and $\beta=e^{i\left(\theta_{j}-\theta_{i}\right)}$, then

$$
-G_{i j}(\lambda)=\frac{a^{1-\lambda}+a^{\lambda-1}+\beta\left(2-a^{1-\lambda}-a^{\lambda-1}\right)-a-a^{-1}}{a-a^{-1}-\left(a^{1-\lambda}-a^{\lambda-1}\right)-\beta\left(a^{\lambda}-a^{-\lambda}\right)}=\frac{-z(\lambda)}{b(\lambda)} .
$$

Observe that $b(\lambda) \underset{\lambda \rightarrow 0}{\longrightarrow} 0$, while $z(\lambda) \underset{\lambda \rightarrow 0}{\longrightarrow} \beta\left(a+a^{-1}-2\right) \neq 0$. Therefore, we have that $G(\lambda)_{i j} \underset{\lambda \rightarrow 0}{\longrightarrow} \infty$.

4. Suppose that $D$ has at least two eigenvalues $d_{i}$ and $d_{j}$ such that $\left|d_{i}\right| \neq\left|d_{j}\right|$. Then, by the description $Q_{D}=\Psi_{J} P_{\mathbb{I m}} \Psi_{J}^{-1}$ given in 5 of Remark 5.1.2, and the fact that $G(\lambda) \in \mathcal{M}_{r}^{a h}(\mathbb{C})$ (which implies that $P_{\mathbb{R e}} \Psi_{G(\lambda)}=\Psi_{G(\lambda)} P_{\mathbb{I m}}$ ), we have that

$$
\left(I-Q_{D}\right) \Psi_{G(\lambda)} Q_{D}=\Psi_{J} P_{\mathbb{R} e} \Psi_{G(\lambda)} P_{\mathbb{I} m} \Psi_{J}^{-1}=\Psi_{J} \Psi_{G(\lambda)} P_{\mathbb{I m}} \Psi_{J}^{-1}
$$


Since $G \in \mathcal{M}_{r}^{a h}(\mathbb{C})$, then $\left\|\Psi_{G(\lambda)}\right\|=\left\|\Psi_{G(\lambda)} P_{\mathbb{I m}}\right\|$. Hence

$$
\left\|P_{D, \lambda}\right\| \geq\left\|\left(I-Q_{D}\right) P_{D, \lambda} Q_{D}\right\|=\left\|\Psi_{G(\lambda)}\right\| \geq\left|G(\lambda)_{i j}\right| \underset{\lambda \rightarrow 0}{\longrightarrow} \infty .
$$

Then the map $\lambda \mapsto P_{D, \lambda}$ can not be constant.

Remark 5.2.2. Note that, using Eq. (5.8), Remark 5.2.1 (particularly Eqs. (5.9) and (5.10) ), and the notations of the Theorem, we can conclude that the map

$$
(0,1) \times \mathcal{U}(D) \ni(\lambda, N) \longmapsto A d_{\sigma_{D}(N)} P_{D, \lambda} A d_{\sigma_{D^{(N)}}}=P_{N, \lambda}
$$

is of class $C^{\infty}$. Another way to prove it is using that the map $(\lambda, N) \mapsto T_{N} \Delta_{\lambda}$ is smooth, and then to apply Eqs. (5.2) and (5.6).

\section{References}

[1] A. Aluthge, On p-hyponormal operators for $0<p<1$, Integral Equations Operator Theory 13 (1990), 307-315.

[2] T. Ando, Aluthge Transforms and the Convex Hull of the Eigenvalues of a Matrix, Linear Multilinear Algebra 52 (2004), 281-292.

[3] T. Ando and T. Yamazaki, The iterated Aluthge transforms of a 2-by-2 matrix converge, Linear Algebra Appl. 375 (2003), 299-309.

[4] J. Antezana, P. Massey and D. Stojanoff, $\lambda$-Aluthge transforms and Schatten ideals, Linear Algebra Appl. 405 (2005), 177-199.

[5] J. Antezana, E. Pujals and D. Stojanoff, Convergence of iterated Aluthge transform sequence for diagonalizable matrices, Advances in Math., to appear. Los Alamos preprint version in www.arxiv.org/abs/math.FA/0604283.

[6] Corach G., Porta H. and Recht L.; The geometry of spaces of projections in $\mathrm{C}^{*}$-algebras, Adv. Math. 101 (1993), 59-77.

[7] Corach G., Porta H. and Recht L.; The geometry of spaces of selfadjoint invertible elements of a $\mathrm{C}^{*}$-algebra, Integral Equations and Operator Theory 16 (1993), 771-794.

[8] J. L. Daleckiŭ, S. G. Kreŭn, Formulas of differentiation according to a parameter of functions of Hermitian operators. (Russian) Doklady Akad. Nauk SSSR (N.S.) 76, (1951). 13-16.

[9] J. L. Daleckiü, S. G. Kreĭn, Integration and differentiation of functions of Hermitian operators and applications to the theory of perturbations. (Russian) Voronež. Gos. Univ. Trudy Sem. Funkcional. Anal. 1956 (1956), no. 1, 81-105.

[10] K. Dykema and H. Schultz, On Aluthge Transforms: continuity properties and Brown measure, preprint. Los Alamos preprint version in the site arXiv:math/0512197v3 [math.OA]

[11] M. W. Hirsch, C. C. Pugh, and M. Shub, Invariant manifolds, Lecture Notes in Mathematics, Vol. 583. Springer-Verlag, Berlin-New York, 1977.

[12] R. Horn y C. Johnson; Topics in Matrix Analysis, Cambridge University Press, Cambridge, 1991.

[13] Huajun Huang and Tin-Yau Tam, On the Convergence of the Aluthge sequence, Math Inequalities and Appl. to appear.

[14] I. Jung, E. Ko, and C. Pearcy, Aluthge transform of operators, Integral Equations Operator Theory 37 (2000), 437-448.

[15] I. Jung, E. Ko, and C. Pearcy, The Iterated Aluthge Transform of an operator, Integral Equations Operator Theory 45 (2003), 375-387. 
[16] K. Okubo, On weakly unitarily invariant norm and the Aluthge Transformation, Linear Algebra and Appl. 371(2003), 369-375.

[17] Porta H. and Recht L.; Minimality of geodesics in Grassmann manifolds, Proc. Amer. Math. Soc., 100, (1987), 464-466.

[18] Porta H. and Recht L.; Variational and convexity properties of families of involutions, Integr. Equat. Oper. Th. 21 (1995), 243-253.

[19] P. Y. Wu, Numerical range of Aluthge transform of operator, Linear Algebra and Appl. 357(2002), 295-298.

[20] T. Yamazaki, An expression of the spectral radius via Aluthge transformation, Proc. Amer. Math. Soc. 130 (2002), 1131-1137. 